\title{
A C-Band Scatterometer Simultaneous Wind/Rain Retrieval Method
}

David G. Long

david_long@byu.edu

Congling Nie

Follow this and additional works at: https://scholarsarchive.byu.edu/facpub

Part of the Electrical and Computer Engineering Commons

\section{Original Publication Citation}

Congling Nie, and D. G. Long. "A C-Band Scatterometer Simultaneous Wind/Rain Retrieval

Method." Geoscience and Remote Sensing, IEEE Transactions on 46.11 (28): 3618-31

\section{BYU ScholarsArchive Citation}

Long, David G. and Nie, Congling, "A C-Band Scatterometer Simultaneous Wind/Rain Retrieval Method" (2008). Faculty Publications. 155.

https://scholarsarchive.byu.edu/facpub/155

This Peer-Reviewed Article is brought to you for free and open access by BYU ScholarsArchive. It has been accepted for inclusion in Faculty Publications by an authorized administrator of BYU ScholarsArchive. For more information, please contact ellen_amatangelo@byu.edu. 


\title{
A C-Band Scatterometer Simultaneous Wind/Rain Retrieval Method
}

\author{
Congling Nie and David G. Long, Fellow, IEEE
}

\begin{abstract}
Using collocated ERS scatterometer (ESCAT), Tropical Rainfall Measuring Mission (TRMM) Precipitation Radar (PR), and European Centre for Medium-Range Weather Forecasts (ECMWF) data, the effects of rain on ESCAT wind-only retrieval are evaluated. Additional scattering from rain causes estimated wind speeds to appear higher than expected. Selected directions of the rain-corrupted wind vectors are biased toward along-track directions under conditions of heavy rain, which is regardless of the true wind direction. Rain becomes more significant for data acquired at a high incidence angle. To compensate for rain-induced backscatter, a simultaneous wind/rain retrieval (SWRR) method, which simultaneously retrieves wind velocity and surface rain rate from ESCAT measurements with an incidence angle $>40^{\circ}$, is developed by using a C-band wind/rain backscatter model. The performance of SWRR under typical wind/rain conditions is evaluated through simulation and validation with collocated TRMM PR and ECMWF data sets. SWRR is shown to significantly improve wind velocity estimates, and the SWRR estimated surface rain rate has relatively high accuracy in moderate to heavy rain cases. Although SWRR-retrieved rain is somewhat biased, it can be corrected. We note that for ESCAT, about $1.5 \%$ of all the collocated measurements are affected by significant rain.
\end{abstract}

Index Terms-Atmospheric measurements, radar cross section, radar meteorological factors.

\section{INTRODUCTION}

$\mathbf{T}$ HE C-BAND ERS scatterometer (ESCAT) of the active microwave instrument on the European Remote Sensing (ERS) satellites (ERS-1 launched in 1991 and ERS-2 in 1995) was designed to measure surface wind speeds and directions over the ocean. The ESCAT signal is traditionally considered transparent to rain. However, recent studies reveal that ESCAT measurements can be adversely affected by moderate to heavy rains at high incidence angles due to the surface effects of rain [1]-[3]. Considering that conventional wind-only retrieval does not explicitly account for the sensitivity of backscatter measurements to rain, rain contamination introduces errors to retrieved wind velocities, particularly at high incidence angles. In order to prevent adverse rain impact on retrieved winds, quality control (QC) methods [4]-[6] are used to discard raincontaminated measurements - a process that causes the loss of

Manuscript received November 21, 2007; revised February 28, 2008. Current version published October 30, 2008.

The authors are with the Department of Electrical and Computer Engineering, Brigham Young University, Provo, UT 84602 USA (e-mail: nie@mers. byu.edu; long@ee.byu.edu).

Color versions of one or more of the figures in this paper are available online at http://ieeexplore.ieee.org.

Digital Object Identifier 10.1109/TGRS.2008.922146 coverage. Considering that the successor of ESCAT, which is the advanced scatterometer instrument (ASCAT) on the MetOpA satellite, has a higher incidence angle coverage than ESCAT, wind retrieval from ASCAT measurements is expected to be more sensitive to rain. Hence, evaluating the influence of rain on ESCAT wind retrieval and developing improved wind retrieval methods are necessary for improving the accuracy and coverage of C-band scatterometer wind estimates in raining areas. Furthermore, rain rate information can be simultaneously estimated [7], which is a side benefit of the new wind retrieval method. Based on the simultaneous wind/rain retrieval (SWRR) method retrieved rain rate, an improved rain flagging method can be developed for ESCAT.

Wind retrieval from scatterometer measurements over the ocean is generally an inversion of the geophysical model function (GMF) which describes $\sigma^{\circ}$, which is the normalized scatterometer backscattering cross section, as a function of wind vector, incidence angle, and polarization. This inversion is generally based on maximum likelihood estimation (MLE). Due to symmetry in the GMF and noise, multiple solutions (defined as "ambiguities") are found when minimizing the MLE cost function. After applying an ambiguity removal algorithm, a unique wind estimate is selected for a specific wind vector cell (WVC).

In raining areas, the $\sigma^{\circ}$ of the ocean's surface is altered by rain. Rain effects include atmospheric and surface effects. Falling raindrops in the atmosphere attenuate and scatter the scatterometer signal. For C-band, rain atmospheric attenuation and scattering is considered negligibly small except for heavy rain. The surface effects of rain are complicated. Raindrops striking the water create various splash products, including rings, stalks, and crowns, which backscatter the scatterometer signal. These splash products have different contributions to the backscattering. Ring waves are the dominant feature for radar backscattering at all incidence angles for VV polarization. Raindrops impinging on the sea surface also generate turbulence in the upper water layer, which attenuates the capillary wave spectrum. The effect of rain on the ocean surface depends on the wavelength of the water waves. For an incidence angle that is higher than $30^{\circ}$, the net effect of rain generally enhances the backscatter [1], [3]. In addition to the modification of the sea surface roughness by the impact of raindrops, the sea surface roughness is also affected by the airflow associated with the rain event. To model these complex rain effects on $\sigma^{\circ}$, a C-band wind/rain backscatter model for ESCAT measurements at incidence angles higher than $40^{\circ}$ is proposed in [1]. The model combines the rain-induced backscatter terms into a single effective rain backscatter, which is described in Section III-A. 
To evaluate the effects of rain on C-band wind-only retrieval, we use collocated wind velocities from the ESCAT windonly retrieval, surface rain rates from the Tropical Rainfall Measuring Mission (TRMM) Precipitation Radar (PR), and numerical predicted wind velocities from the European Center for Medium-Range Weather Forecasts (ECMWF). The ESCAT data and TRMM PR measurements are collocated within \pm 15 min. About 82181 collocations are collected from a period of 16 months between August 1, 1999 and December 31, 2000. To ensure the quality of the analysis, only the collocations in which the overlapping PR swath contains more than $2.5 \%$ of the measurements flagged as rain-certain in the TRMM level 2A25 data are used in this paper. The ECMWF-predicted wind fields are trilinearly interpolated in space and time to the ESCAT data times and locations. The spatial resolution of the ECMWF winds is $1^{\circ} \times 1^{\circ}$ latitude-longitude, with a temporal resolution of $6 \mathrm{~h}$.

To compensate for rain-induced backscatter, we apply the C-band wind/rain backscatter model [1] in the wind retrieval process, developing a SWRR method for ESCAT. The method simultaneously retrieves surface rain rates and wind vectors, using an adjusted MLE. Considering that the backscatter model is only usable at an incidence angle $>40^{\circ}$, we implement SWRR for data at WVC 13-19. Conventional wind-only retrieval is used at low incidence angles.

The organization of this paper is as follows. In Section II, the degradation of the rain on the wind-only wind retrieval results is evaluated and analyzed. Following that, we describe the methodology of SWRR in Section III. The normalized standard deviation (STD) of rain-induced backscatter is estimated in Section IV. In Sections V and VI, we evaluate the performance of SWRR by simulation and with the use of the collocated data set. In Section VII, a case study is presented. Conclusions are reached in Section VIII.

\section{RAIN EFFECTS ON WIND-ONLY RETRIEVAL}

In this section, we briefly describe the background of conventional wind-only retrieval and analyze the impact of the rain on the wind retrieval procedure by simulation. We then evaluate the real rain effect on wind-only retrieval using the collocated data set.

\section{A. Conventional Wind-Only Retrieval}

For ESCAT, the wind-only retrieval process involves the inversion of the GMF given $\sigma^{\circ}$ triplet measurements. Here, the wind-only GMF inversion method is based on the minimization of a MLE cost function, assuming Gaussian noise and independent samples

$$
\operatorname{MLE}(z \mid s, d)=\sum_{i=1}^{3} \frac{\left(\sigma_{i}^{\circ}-\mathbf{M}\left(s, d, \phi_{i}, \theta_{i}\right)\right)^{2}}{\left(\varsigma_{i}(s, d)\right)^{2}}
$$

where $\sigma_{i}^{\circ}$ is the measured $\sigma^{\circ}$ value, $\mathbf{M}$ is the GMF, $s$ is the wind speed, $d$ is the wind direction, $\phi_{i}$ is the azimuth angle of the radar beam, and $\theta_{i}$ is the incidence angle of the radar beam. The index $i$ indicates antenna beam position. $\left(\varsigma_{i}\right)^{2}$ is the variance of measurements, which is a measure of the noise in the $\sigma^{\circ}$ measurements. The variance $\left(\varsigma_{i}\right)^{2}$ is affected by many factors, including uncertainty in GMF, signal noise due to fading, thermal noise, and beam-filling effects. It can be expressed as $\varsigma_{i}(s, d)=K_{p} \mathbf{M}\left(s, d, \phi_{i}, \theta_{i}\right)$, where $K_{p}$ is the normalized STD of the measurements. $K_{p}$ can be expressed as a combination of $K_{p m}$, the normalized STD of GMF, and $K_{p c}$, which is the normalized STD of the communication or signal noise [8]

$$
K_{p}=\sqrt{K_{p c}^{2}+K_{p m}^{2}+K_{p c}^{2} K_{p m}^{2}}
$$

In general, the term $K_{p c}$ has the form

$$
K_{p c}=\sqrt{\alpha+\frac{\beta}{\sigma_{t}}+\frac{\gamma}{\sigma_{t}^{2}}}
$$

where $\alpha, \beta$, and $\gamma$ are coefficients that depend on the fading characteristics of the surface scatterers and the signal-to-noise ratio (SNR) at the receiver [8], [9] and $\sigma_{t}$ is the true $\sigma^{\circ}$ of the ocean surface without communication noise. Considering that the measurement SNR for ESCAT is very high, $K_{p c}$ is small compared with the geophysical-modeling error $K_{p m}$ [10]. Lacking an accurate model for $K_{p c}$ coefficients, we assume $K_{p c}$ to be constant for ESCAT, which is typically 0.05 . The geophysical-modeling error $K_{p m}$ is caused by many factors. One of them is the uncertainty of GMF, considering that the empirical GMF is not an exact relationship. Many parameters that are not included in the GMF, such as local salinity, temperature, and long waves, can change the observed $\sigma^{\circ}$ for a fixed wind velocity. Beam filling, which is due to the wind variability within the resolution cell and the nonuniform spatial averaging that is inherent in the radar measurements is another factor for $K_{p m}$ [7], [11].

The minimization of the MLE results in one to four local minima (ambiguities), which represent possible wind vector solutions. The two primary ambiguities correspond to the two most likely solutions, differing by about $180^{\circ}$ in direction. The occurrence and location of the other ambiguities often depend on the normalization [11]. A method proposed by Stoffelen and Anderson [11] transforms the measurements to a $z$ space by the form $z=\left(\sigma^{\circ}\right)^{0.625}$ and results in a circular distribution that is ideal for inversion.

Due to multiple ambiguities, an ambiguity removal procedure must be implemented to choose one unique solution. The ambiguity removal procedure uses median filtering and nudging techniques to choose the best solution. For the ESCAT windonly retrieval, a selection filter is implemented to iteratively select the ambiguity at each WVC, which is based on a weighted average of the differences from the surrounding WVCs. At each WVC, the selection filter is nudged by the ECMWF model "first guess at appropriate time" wind vectors [11].

\section{B. Analysis of Rain on Wind Retrieval}

As mentioned in Section I, rain surface backscatter can dominate the total backscatter under conditions of low-to-medium wind speed and heavy rain at high incidence angles. Using 

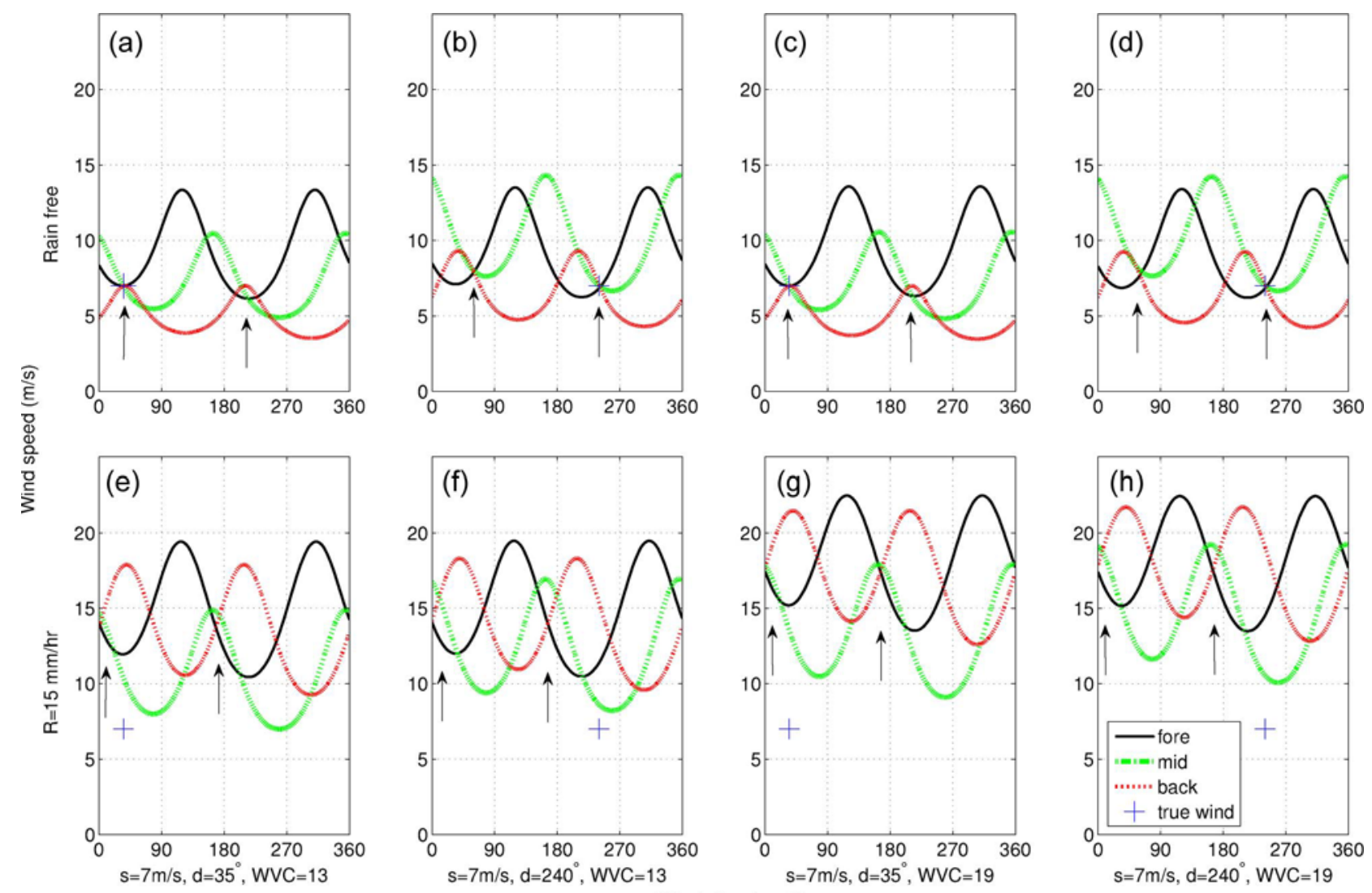

Fig. 1. Visualization of the wind retrieval procedure for data in WVCs (left panel) 13 ( $\theta_{\text {fore } / \text { aft }} \approx 48.6^{\circ}$ and $\theta_{\text {mid }} \approx 37.7^{\circ}$ ) and (right panel) $19\left(\theta_{\text {fore } / \text { aft }} \approx\right.$ $56.6^{\circ}$ and $\theta_{\text {mid }} \approx 45.4^{\circ}$ ). The intersections of the three curves correspond to the true wind velocity and aliases. The arrows in the plots point to the two major ambiguities. (a)-(d) Wind retrieval procedure for rain-free data. (e)-(h) Procedure for rain-corrupted data. The true wind speed is $7 \mathrm{~m} / \mathrm{s}$. The true wind directions are $35^{\circ}$ and $240^{\circ}$. The true wind vector is shown as a "+" symbol in the plots. The true rain rate is $31.6 \mathrm{~mm} / \mathrm{h}$. When the backscatter is dominated by rain, intersections tend to be associated with along-track directions.

the C-band wind/rain backscatter model described in (4)-(6), we show how rain affects the conventional wind-only wind retrieval process by simulation under typical low wind and heavy rain cases for WVCs with high incidence angles. To visualize the wind retrieval procedure, we use the locus of wind velocities that give rise to a single $\sigma^{\circ}$ for fixed radar incidence and azimuth angles. Fig. 1 shows a plot of $\sigma^{\circ}$ for three sets of radar angles that are representative of simulated data without rain [in Fig. 1(a)-(d)] and with rain [in Fig. 1(e) and (f)] from the ESCAT scatterometer for WVCs 13 and 19. These plots are generated by choosing a wind speed and direction and calculating $\sigma^{\circ}$ values using the wind/rain backscatter model. For each set of radar incidence and azimuth angle, all the wind speeds and directions that induce the specific $\sigma^{\circ}$ are plotted as a single curve.

As shown in Fig. 1(a)-(d), the three measurements without noise from the fore, middle, and aft beams have an intersection corresponding to the true wind velocity. Due to upwind/ downwind similarity, there is a near intersection point of about $180^{\circ}$ from the true wind direction. In the presence of rain, the magnitude of backscatter increases and tends toward isotropic. Because of the viewing geometry of ESCAT measurements under the condition of heavy rain, the two primary minima tend to be at approximately $180^{\circ} \pm 14^{\circ}$ and $360^{\circ} \pm 14^{\circ}$ directions,

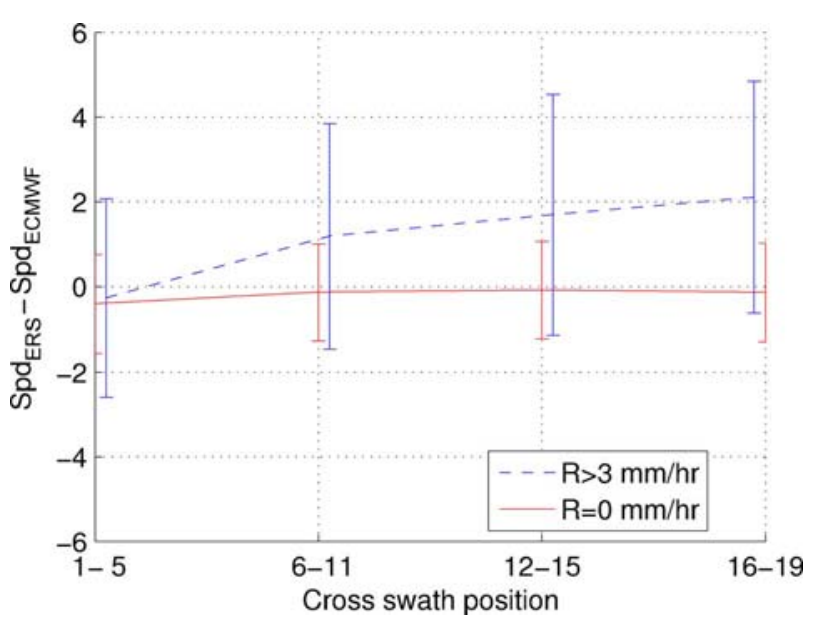

Fig. 2. Mean of the difference between the wind-only retrieval method selected wind speed ambiguity and collocated ECMWF wind speed under rainfree conditions and rain rate of over $3 \mathrm{~mm} / \mathrm{h}$ for different WVC bins. Error bar in the figure represents the STD.

which is regardless of the true wind speed. These directions correspond to the along-swath direction, as shown in Fig. 1(e)-(h). Fig. 1(b) also shows that the wind speeds corresponding to the intersections are biased high. Comparing the plots for WVC 13 
TABLE I

INCIDENCE ANGLES (IN DEGREE) OF Fore/AFT (F/A) AND Mid (M) ANTENNAS Of THE 19 WVCS

\begin{tabular}{|c|c|c|c|c|c|c|c|c|c|c|c|c|c|c|c|c|c|c|}
\hline$\overline{W V C} 1$ & 2 & 3 & 4 & 5 & 6 & 7 & 8 & 9 & 10 & 11 & 12 & 13 & 14 & 15 & 16 & 17 & 18 & 19 \\
\hline$\overline{F / A} 25.1$ & 27.5 & 29.8 & 32.0 & 34.2 & 36.3 & 38.2 & 40.2 & 42.0 & 43.7 & 45.4 & 47.0 & 48.6 & 50.0 & 51.5 & 52.8 & 54.1 & 55.4 & 56.6 \\
\hline 18.2 & 20.0 & 21.8 & 23.6 & 25.3 & 27.0 & 28.7 & 30.3 & 31.9 & 33.4 & 34.9 & 36.3 & 37.7 & 39.1 & 40.4 & 41.7 & 42.9 & 44.2 & 45.4 \\
\hline
\end{tabular}

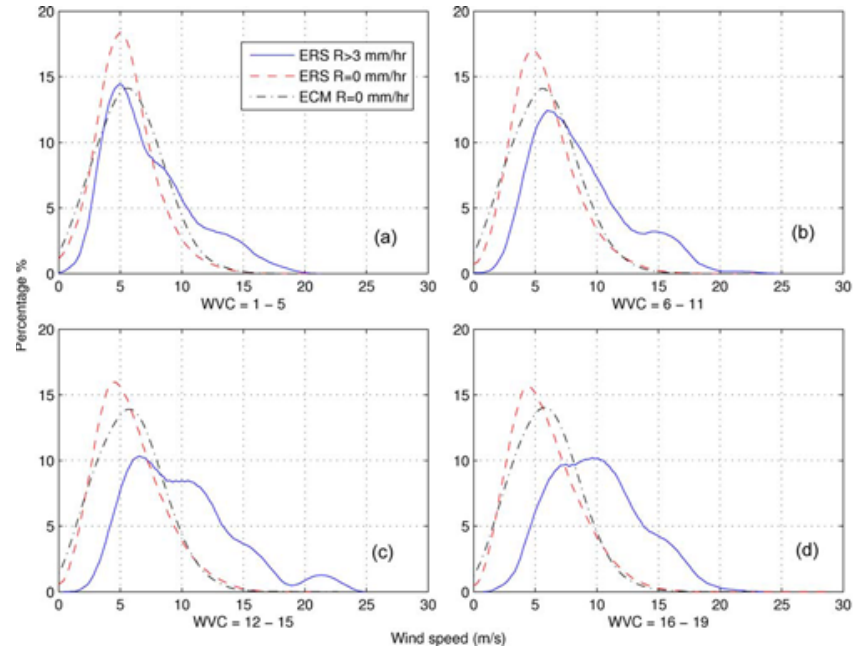

Fig. 3. Normalized histogram of the wind-only retrieval selected wind speed ambiguities for rain-free conditions (ERS $R=0 \mathrm{~mm} / \mathrm{h}$ ) and rain rate of over $3 \mathrm{~mm} / \mathrm{h}$ (ERS $R>3 \mathrm{~mm} / \mathrm{h}$ ) compared with collocated ECMWF wind speeds (ECM $R=0 \mathrm{~mm} / \mathrm{h}$ ) for WVC bins (a) 1-5, (b) 6-11, (c) 12-15, and (d) 16-19.

(with incidence angle $48.5^{\circ}$ for the fore and aft beams and $37.7^{\circ}$ for the midbeam) with WVC 19 (with incidence angle $57^{\circ}$ for the fore and aft beams and $45^{\circ}$ for the midbeam), the speed bias becomes more serious for higher incidence angles. Similar results are found for rain-corrupted wind estimates from Ku-band scatterometers [12], [13], although the direction estimate aligns cross-track in this case. The different wind direction features of the rain-corrupted estimates from the two scatterometers are mainly due to the different antenna viewing geometries of the two instruments.

\section{Rain Effects on Wind-Only Vectors}

After a brief theoretical analysis, we evaluate the effect of rain on the wind-only retrieved wind estimates using the collocated data set. To illustrate the influence of rain, we investigate the statistics of the wind speed and the wind direction retrieved from rain-free and rain-corrupted $\sigma^{\circ}$ measurements for different WVC positions and rain rate ranges. Fig. 2 shows the mean of the difference between the selected wind speed ambiguity and the collocated ECMWF wind speed $\left(\operatorname{Spd}_{\mathrm{ERS}}-\mathrm{Spd}_{\mathrm{ECMWF}}\right)$ for rain-free and rain-corrupted (rain rate $>3 \mathrm{~mm} / \mathrm{h}$ ) cases at WVCs of 1-5, 6-11, 12-15, and 16-19. As a reminder, the incidence angles of triplet measurements increase with the WVC number. The typical incidence angles of the fore/aft antenna (fore and aft antennas have the same nominal incidence angle) and the midantenna of the WVCs are listed in Table I. The selected wind speeds have no obvious bias under the rain-free condition. Under moderate-to-heavy rains, the selected wind speeds are biased high, and the bias in the wind speed increases from low to high WVC, revealing that the rain impact on wind speed estimation becomes more significant with increasing incidence angle.

We compare the normalized histograms of the selected wind speed and wind direction for rain-free and rain-corrupted conditions and the ECMWF wind speed for rain-free data in Figs. 3 and 4(a) for the same WVCs. The normalized histograms of the selected and the ECMWF wind speeds are consistent for the rain-free conditions. The speed densities of the raincorrupted data gradually shift to the right from WVC with low to high incidence angles, which are consistent with the results in Fig. 2. For wind directions, the histogram of the rain-free selected wind directions agrees well with the collocated ECMWF wind direction. With the presence of rain, peaks develop, revealing that the rain-corrupted wind directions are gradually biased toward the along-track direction. The along-track direction bias becomes more serious with increasing incidence angles.

To evaluate the performance of wind-only MLE, we show the normalized histogram of the wind ambiguity closest to the collocated ECMWF wind for the same rain conditions and WVC. We plot the normalized histogram of the wind direction ambiguity closest to the collocated ECMWF wind for rain-free and rain-corrupted cases at WVCs of 1-5, 6-11, 12-15, and 16-19 in Fig. 4(b). For rain-corrupted measurements at WVCs with high incidence angles, most of the closest wind direction ambiguities align with the along- or crosstrack directions. For these cases, wind-only MLE cannot retrieve the correct wind direction. Hence, a new wind retrieval method must be developed to compensate for the influence of rain.

\section{SWRR}

As we have shown, conventional wind-only retrieval can be adversely affected by moderate-to-heavy rains at high incidence angles. To compensate for rain-induced backscatter, a SWRR method for ESCAT is developed in this section.

\section{A. Methodology}

The SWRR method is based on a simple additive wind/rain backscatter model proposed in [1] and [7], which represents the rain-modified measured backscatter $\sigma_{\mathrm{m}}$ as

$$
\sigma_{\mathrm{m}}=\sigma_{\mathrm{wind}} \alpha_{\mathrm{atm}}+\sigma_{\mathrm{eff}}
$$

where $\sigma_{\mathrm{m}}$ is the ESCAT-measured $\sigma^{\circ}, \sigma_{\text {wind }}$ is the windinduced surface backscatter, $\alpha_{\mathrm{atm}}$ is the two-way rain-induced atmospheric attenuation, and $\sigma_{\text {eff }}$ is the effective rain backscatter due to attenuated surface perturbation and rain-induced atmospheric scattering [1], [7], [22], [23]. $\alpha_{\text {atm }}$ and $\sigma_{\text {eff }}$ are 

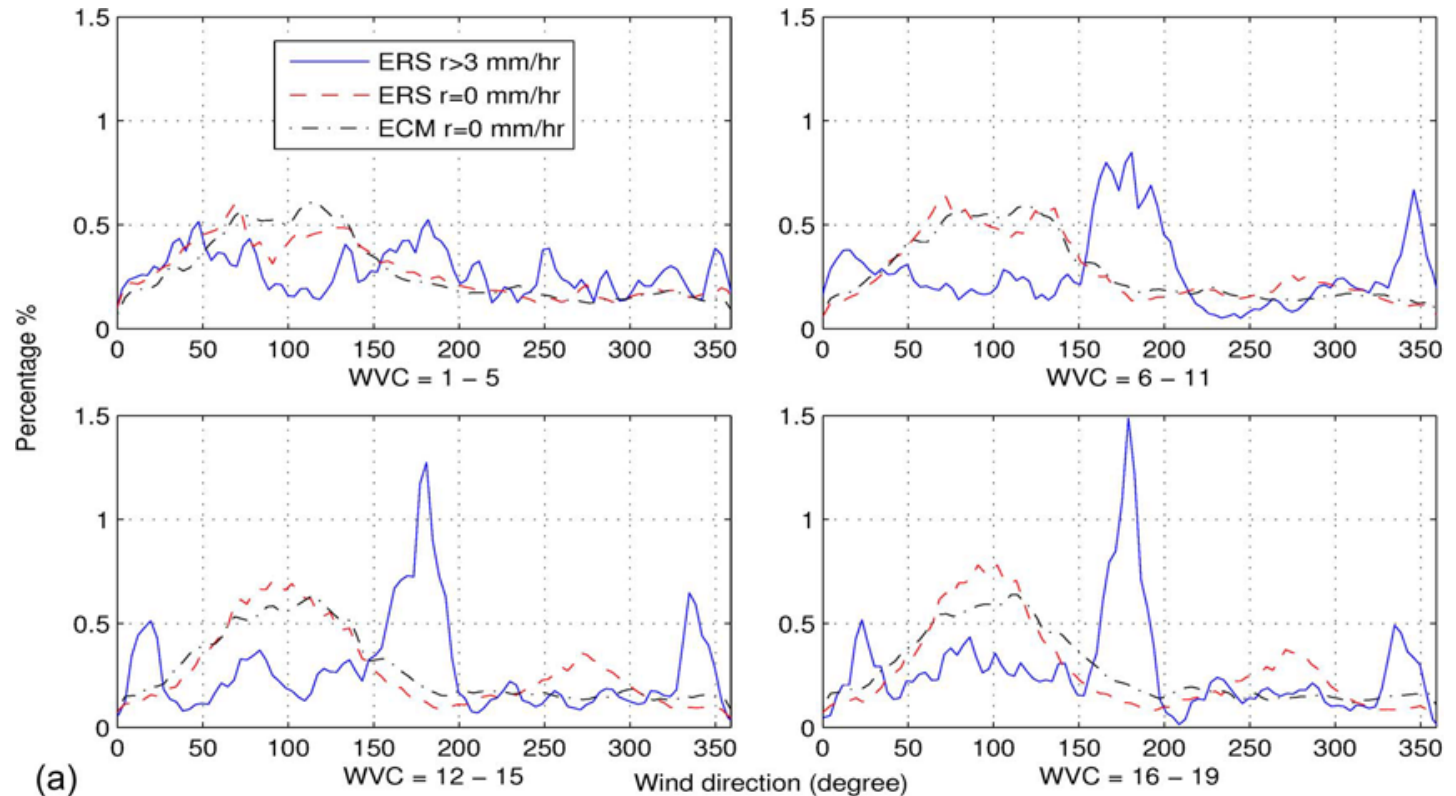

(a)

WVC $=12-15 \quad$ Wind direction (degree)
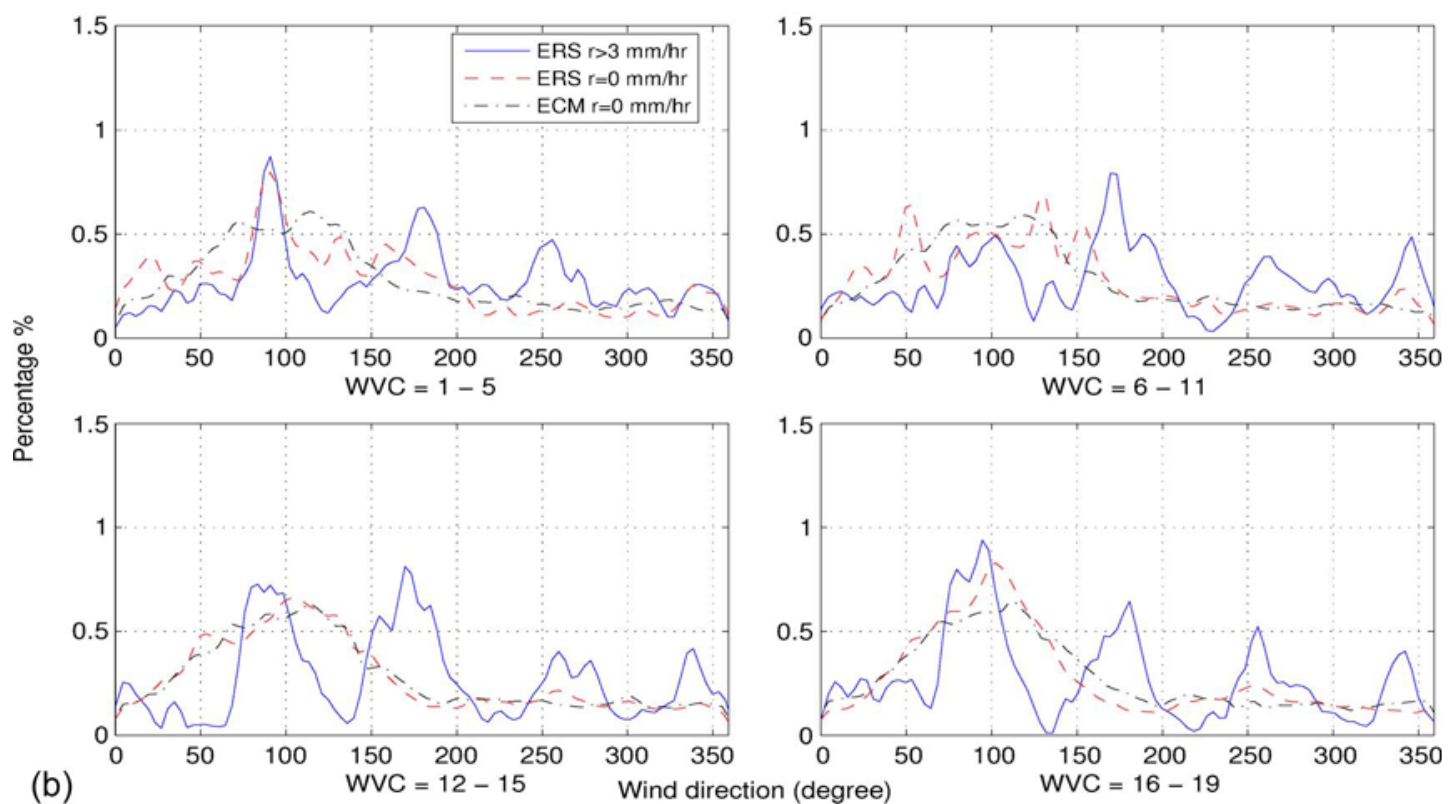

Fig. 4. Normalized histogram of the (a) wind-only retrieval selected wind directions and the (b) direction ambiguities that are closest to the collocated ECMWF wind directions for rain-free conditions (ERS $R=0 \mathrm{~mm} / \mathrm{h}$ ) and rain rate of over $3 \mathrm{~mm} / \mathrm{h}$ (ERS $R>3 \mathrm{~mm} / \mathrm{h}$ ) compared with collocated ECMWF wind directions (ECM $R=0 \mathrm{~mm} / \mathrm{h}$ ) for different $\mathrm{WVC}$ bins.

related to the surface rain rate $R$ in millimeters per hour by empirically derived linear or quadratic $\log -\log$ models [1] as

$$
\begin{aligned}
10 \log _{10}\left(-10 \log _{10} \alpha_{\mathrm{atm}}(\theta)\right) & =10 \log _{10}\left(\operatorname{PIA}_{\mathrm{atm}}(\theta)\right) \\
& =\sum_{n=0}^{N} x_{a}(n) R_{\mathrm{dB}}^{n} \\
10 \log _{10}\left(\sigma_{\mathrm{eff}}(\theta)\right) & =\sum_{n=0}^{N} x_{e}(n) R_{\mathrm{dB}}^{n}
\end{aligned}
$$

where $R_{\mathrm{dB}}=10 \log _{10}(R)$ and where $x_{a}(n)$ and $x_{e}(n)$ are the corresponding model coefficients. $N=1$ for the linear model, and $N=2$ for the quadratic model. The coefficients of the power law models of $\alpha_{\text {atm }}$ and $\sigma_{\text {eff }}$ are given in Tables II and III, respectively.
Applying the rain model with the conventional wind GMF function CMOD5, the wind/rain GMF is

$$
\sigma^{\circ}=\operatorname{CMOD} 5(s, d, \theta, \phi) \alpha_{\text {atm }}(R, \theta)+\sigma_{\text {eff }}(R, \theta) .
$$

Then, the MLE likelihood function of (1) is written as

$$
\operatorname{MLE}^{\prime}(z \mid s, d, R)=\sum_{i=1}^{3} \frac{\left(\sigma_{i}^{\circ}-\mathbf{M}^{\prime}\left(s, d, \phi_{i}, \theta_{i}, R\right)\right)^{2}}{\left(\varsigma_{i}^{\prime}(s, d)\right)^{2}}
$$

where $\mathbf{M}^{\prime}$ is the wind/rain GMF in the equations. $\left(\varsigma^{\prime}\right)^{2}$ is the variance of the rain-contaminated measurement, which is estimated in the next section. The wind velocity and rain rate estimates are simultaneously retrieved by minimizing the new 
TABLE II

MOdel COEFFICIENTS (LiNEAR AND QUADRATIC) of PIA $\operatorname{Patm}(\theta)$

\begin{tabular}{ccccc}
\hline$\theta\left({ }^{\circ}\right)$ & & $x_{a}(0)$ & $x_{a}(1)$ & $x_{a}(2)$ \\
\hline \multirow{2}{*}{$40-44$} & Linear & -18.23 & 1.25 & \\
\cline { 2 - 5 } & Quadratic & -18.18 & 1.25 & -0.00060 \\
\hline \multirow{2}{*}{$44-49$} & Linear & -17.89 & 1.25 & \\
\cline { 2 - 5 } & Quadratic & -17.79 & 1.24 & -0.0016 \\
\hline \multirow{2}{*}{$49-53$} & Linear & -17.44 & 1.26 & \\
\cline { 2 - 5 } & Quadratic & -17.39 & 1.25 & -0.00081 \\
\hline $53-57$ & Linear & -17.12 & 1.25 & \\
\cline { 2 - 5 } & Quadratic & -17.05 & 1.24 & -0.0012 \\
\hline
\end{tabular}

TABLE III

Model CoefFicIEnTs (LiNEAR AND QuAdRATIC) OF $\sigma_{\text {eff }}(\theta)$

\begin{tabular}{ccccc}
\hline$\theta\left({ }^{\circ}\right)$ & & $x_{\mathrm{e}}(0)$ & $x_{\mathrm{e}}(1)$ & $x_{\mathrm{e}}(2)$ \\
\hline \multirow{3}{*}{$40-44$} & Linear & -27.21 & 0.703 & \\
\cline { 2 - 5 } & Quadratic & -27.60 & 0.728 & 0.0016 \\
\hline \multirow{2}{*}{$44-49$} & Linear & -27.37 & 0.759 & \\
\cline { 2 - 5 } & Quadratic & -27.61 & 0.76 & 0.0030 \\
\hline \multirow{2}{*}{$49-53$} & Linear & -27.87 & 0.797 & \\
\cline { 2 - 5 } & Quadratic & -27.96 & 0.768 & 0.0034 \\
\hline $53-57$ & Linear & -28.19 & 0.851 & \\
\cline { 2 - 5 } & Quadratic & -28.78 & 0.791 & 0.0109 \\
\hline
\end{tabular}

MLE for $s, d$, and $R$ given the triplet $\sigma^{\circ}$ measurements. For simplicity, the normalization method proposed in [11] is not applied in this paper, although the normalization method may further improve the accuracy of SWRR. Similar to the windonly retrieval method, the minimization of the SWRR MLE results in multiple ambiguities with the corresponding wind speed, wind direction, and surface rain rates. Here, we use an adjusted median filter-based method based on the method proposed in [24] to select a final estimate. The adjusted median filter weighs each ambiguity by the exponential of its likelihood value. Collocated ECMWF wind fields are used for nudging.

\section{B. Variance Model}

Before deriving the variance model of the rain-contaminated measurements, some assumptions are required. The noise in the measurement is assumed to be white Gaussian noise. The communication noise $K_{p c}$ and wind-only GMF uncertainty $K_{p m}$ are assumed unchanged under raining conditions. $K_{p m}$, $K_{p a}$ (the uncertainty of $\alpha_{\text {atm }}$ ), and $K_{p e}$ (the uncertainty of $\left.\sigma_{\text {eff }}\right)$ are assumed to be independent. Under these assumptions, the noisy wind/rain backscatter measured by the scatterometer instrument can be modeled as

$$
\begin{aligned}
\sigma_{\text {meas }}=\left[\sigma_{\text {wind }}\left(1+v_{1} K_{p m}\right) \alpha_{\text {atm }}\left(1+v_{2} K_{p a}\right)\right. & \\
& \left.+\sigma_{\text {eff }}\left(1+v_{3} K_{p e}\right)\right]\left(1+K_{p c} v_{4}\right)
\end{aligned}
$$

where $\sigma_{\text {wind }}$ can be approximately represented by the windonly GMF M. $v_{1}, v_{2}, v_{3}$, and $v_{4}$ are zero-mean Gaussian random variables. Considering that $K_{p a}$ is negligible compared with $K_{p e}$ [14], $K_{p a}$ is ignored in the derivation of $\operatorname{Var}\left(\sigma_{\text {meas }}\right)$. The variance of the measured $\sigma^{\circ}$, which is $\operatorname{Var}\left(\sigma_{\text {meas }}\right)$, is

$$
\begin{aligned}
\operatorname{Var}\left(\sigma_{\text {meas }}\right) \approx\left(1+K_{p c}^{2}\right)\left(\mathbf{M}^{2} \alpha_{\text {atm }}^{2} K_{p m}^{2}+\sigma_{\text {eff }}^{2} K_{p e}^{2}\right) \\
+K_{p c}^{2}\left(\sigma_{\text {eff }}+\mathbf{M} \alpha_{\text {atm }}\right)^{2} .
\end{aligned}
$$

$K_{p c}$ is available for each WVC in the ESCAT product and is about $0.05 . K_{p m}$ is considered insignificant except at low wind speeds [11], [15]. The $K_{p e}$ term for ESCAT has never been studied but is analyzed and estimated in the following section.

\section{Estimation of $K_{p e}$}

Many factors contribute to $K_{p e}$, including uncertainty in the rain backscatter model and variability caused by the nonuniform beam-filling (NUBF) effect. The value of $K_{p e}$ can be roughly estimated in the range of $0.38-0.45$, which is from the validation of the wind/rain backscatter model, where the normalized STDs due to uncertainty in the model are 1.4 and $1.6 \mathrm{~dB}$ for $\theta=40^{\circ}-49^{\circ}$ and $\theta=49^{\circ}-57^{\circ}$ [1], respectively. However, this value of $K_{p e}$ may be overestimated due to the variability of the temporal collocation, the inherent uncertainty of the ECMWF-predicted winds, and the errors in the TRMM PR-estimated surface rain rates. In this section, we first evaluate the contribution to $K_{p e}$ by the NUBF effect. Then, we adopt a practical method to seek an optimal $K_{p e}$ for the wind/rain retrieval process. Finally, the impact of the value of $K_{p e}$ on the wind/rain retrieval process is investigated.

\section{A. $K_{p e}$ Due to the Beam-Filling Effect}

The size of a typical rain cell is on the order of $5 \mathrm{~km} \mathrm{[25],}$ which is relatively small compared with the $50-\mathrm{km}$-sized 3-dB spatial response function of ESCAT. Hence, the variability due to NUBF is a considerable factor for $K_{p e}$. To estimate the NUBF-induced variability, we estimate the STD of the normalized error between the model-predicted rain effective backscatter $\sigma_{e}^{m}$ calculated from the antenna-weighted-average TRMM PR rain rate and the ESCAT-observed effective backscatter $\sigma_{e}^{e}$. $\sigma_{e}^{e}$ can be approximately estimated by averaging the ESCATobserved effective backscatter $\sigma_{e}^{\prime}$ at each TRMM PR cell in the ESCAT footprint over the 3-dB spatial response function of ESCAT, i.e.,

$$
\sigma_{e}^{e}=\frac{\sum_{i=1}^{N} G(i) \sigma_{e}^{\prime}(i)}{\sum_{i=1}^{N} G(i)}
$$

where $G(i)$ is the ESCAT spatial response function gain at the $i$ th PR measurement and $N$ is the number of PR data points within the ESCAT 3-dB antenna pattern contour.

$\sigma_{e}^{\prime}$ is estimated by projecting the TRMM PR surface rain rate through a rain backscatter model that is unweighted by the spatial response function of ESCAT. The model has the same form as (4) but has different coefficients from the weighted rain backscatter model in [1]. To estimate the coefficients of the unweighted model, we estimate $\sigma_{e}^{e}$ for varying values of the model coefficients of the unweighted model. Comparing $\sigma_{e}^{e}$ with the corresponding $\sigma_{e}^{m}$, we choose the model coefficients that yield the lowest root mean-square (rms) error overall. An estimate of $K_{p e}$ is obtained by taking the STD of the normalized error between $\sigma_{e}^{m}$ and $\sigma_{e}^{e}$

$$
K_{p e} \cong \operatorname{STD}\left(\frac{\sigma_{e}^{e}-\sigma_{e}^{m}}{\sigma_{e}^{m}}\right)
$$




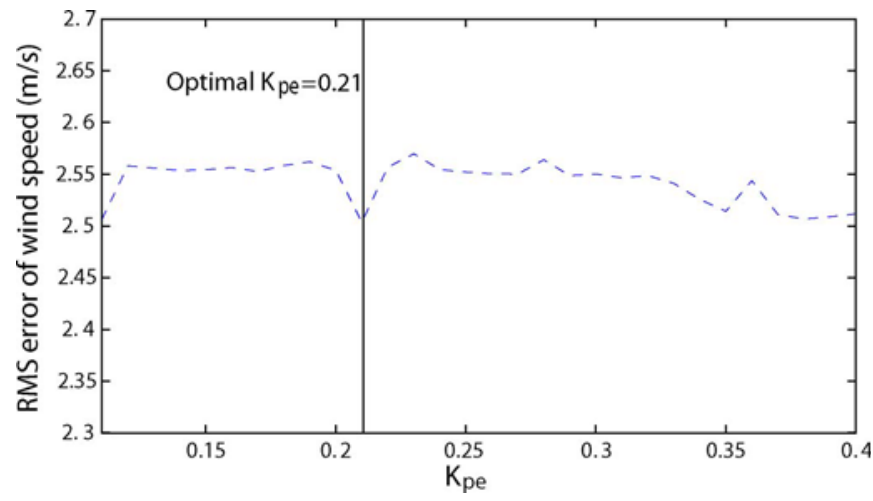

Fig. 5. RMS error between SWRR-retrieved and ECMWF wind speeds as a function of $K_{p e}$ for rain rate $>0 \mathrm{~mm} / \mathrm{h}$. The optimum value of $K_{p e}$ is indicated.

for all surface rain rate observations that are above a rain rate threshold.

The resulting value is $K_{p e} \approx 0.07$ for the rain rate $\geq$ $0.8 \mathrm{~mm} / \mathrm{h}$ and $K_{p e} \approx 0.19$ for the rain rate $<0.8 \mathrm{~mm} / \mathrm{h}$, showing that the model uncertainty due to the beam-filling effect is more significant for low rain rates than for moderate-tohigh rain rates. We note that for relatively large footprints and moderate-to-high rain rates, rain fields often appear relatively uniform. Moreover, the shape of the rain backscatter model function is relatively flat. For these reasons, the model variability due to the beam-filling effect is relatively small for moderate-to-high rain rates.

\section{B. Seeking Optimal $K_{p e}$}

As mentioned earlier, many factors contribute to the model uncertainty. Analyzing all of them is beyond the scope of this paper. Here, we adopt a method proposed in [13] to seek an optimal value of $K_{p e}$ for the wind retrieval process. From (3), we know that the value of $K_{p e}$ can affect the MLE. Hence, the wind/rain estimation is affected by the value of $K_{p e}$. The optimal $K_{p e}$-seeking method is to find the value of $K_{p e}$ for SWRR that yields the best wind speed estimates using the collocated data set. To find the optimal value of $K_{p e}$, we perform SWRR for varying values of $K_{p e}$. The rms wind speed errors between SWRR-retrieved and ECMWF-predicted winds for each $K_{p e}$ are evaluated over the collocated data set.

The wind speed bias between wind-only retrieved winds with CMOD5 and ECMWF winds is about $-0.2 \mathrm{~m} / \mathrm{s}$ [16], which is compensated for in the computation of the rms wind speed error. When $K_{p e}=0.21$, the rms error is at a minimum for measurements with rain rate $>0 \mathrm{~mm} / \mathrm{h}$; hence, we choose this value for $K_{p e}$.

To understand the impact of $K_{p e}$ on SWRR, we show the rms error of the wind speed retrieval in Fig. 5. The STD of the rms wind speed error for various $K_{p e}$ is $0.0191 \mathrm{~m} / \mathrm{s}$, showing that the impact of $K_{p e}$ on SWRR is insignificant.

With an estimated $K_{p e}$, we have all the parameters for SWRR. To validate and evaluate the performance of SWRR, we use both simulation and real collocated data sets, which are illustrated in the following sections.
TABLE IV

Wind SpeEds, Wind DiRECTIONS, AND RAin RATES FOR Simulations

\begin{tabular}{lcccccc}
\hline Wind Speeds $(\mathrm{m} / \mathrm{s})$ & 4 & 8 & 12 & 16 & 20 & 24 \\
Wind Directions (degree) & 0 & 20 & 40 & $\ldots$ & 340 & \\
Rain rates $(\mathrm{mm} / \mathrm{hr})$ & 0 & 1 & 3 & 10 & 30 & \\
\hline
\end{tabular}

\section{Simulations}

In this section, we evaluate the performance of SWRR under various wind/rain conditions by simulation. To achieve this goal, SWRR and wind-only retrieval are performed by using synthetic $\sigma^{\circ}$ measurements created by Monte Carlo simulation under specific wind/rain conditions for WVCs 13, 15, 17, and 19.

\section{A. Simulation Method}

To create simulated $\sigma^{\circ}$ measurements, we project the various wind speeds, wind directions, and rain rates through the wind/rain backscatter model in (4) with typical measurement geometries at each ESCAT WVC. Zero-mean Gaussian random noise with the variance given in (10) is added to $\sigma^{\circ}$. The wind speeds, wind directions, and rain rates used for simulations are listed in Table IV. For each condition, 500 noise realizations are created for each case. After performing SWRR and wind-only retrieval over the realizations, the ambiguities closest to the true wind vectors are selected.

\section{B. Results and Analysis}

To aid in analyzing the results from the simulations, we introduce a parameter, which is the rain ratio $\tau$ [1], [7], to indicate how severely the rain alters the backscatter. The rain ratio shows the average percentage of the rain-induced backscatter that is relative to the total effective backscatter $\sigma_{t}$

$$
\tau=\frac{1}{k} \sum_{l=1}^{k} \frac{\sigma_{e}^{l}}{\sigma_{t}^{l}}
$$

where $k$ is the number of measurements and $\sigma_{e}$ is the raininduced effective backscatter. As in [1], we define three distinct regimes using $\tau$. In regime $1(\tau<0.25)$, wind-induced backscatter dominates and rain information cannot be accurately estimated from the measurements. Wind- and raininduced backscatters are on the same order of magnitude in regime $2(0.25 \leq \tau \leq 0.75)$, and wind and rain information can be simultaneously estimated. In regime 3 , rain-induced backscatter dominates the total backscatter $(\tau>0.75)$; hence only the rain rate can be accurately retrieved.

To compare the performance of SWRR and wind-only retrieval under varying regimes, we show the mean and STD of the difference between SWRR and wind-only retrieved and true wind speed $\operatorname{Err}_{s}=$ Speed $_{\text {retrieved }}-$ Speed $_{\text {true }}$ for a typical wind and rain case in each regime for WVCs 13, 15, 17, and 19 in Fig. 6(a). Due to the varying incidence angles at different WVCs, the value of $\tau$ varies at different WVCs under the same wind and rain conditions. The corresponding true wind speed is $8 \mathrm{~m} / \mathrm{s}$, which is close to the mean wind speed over 

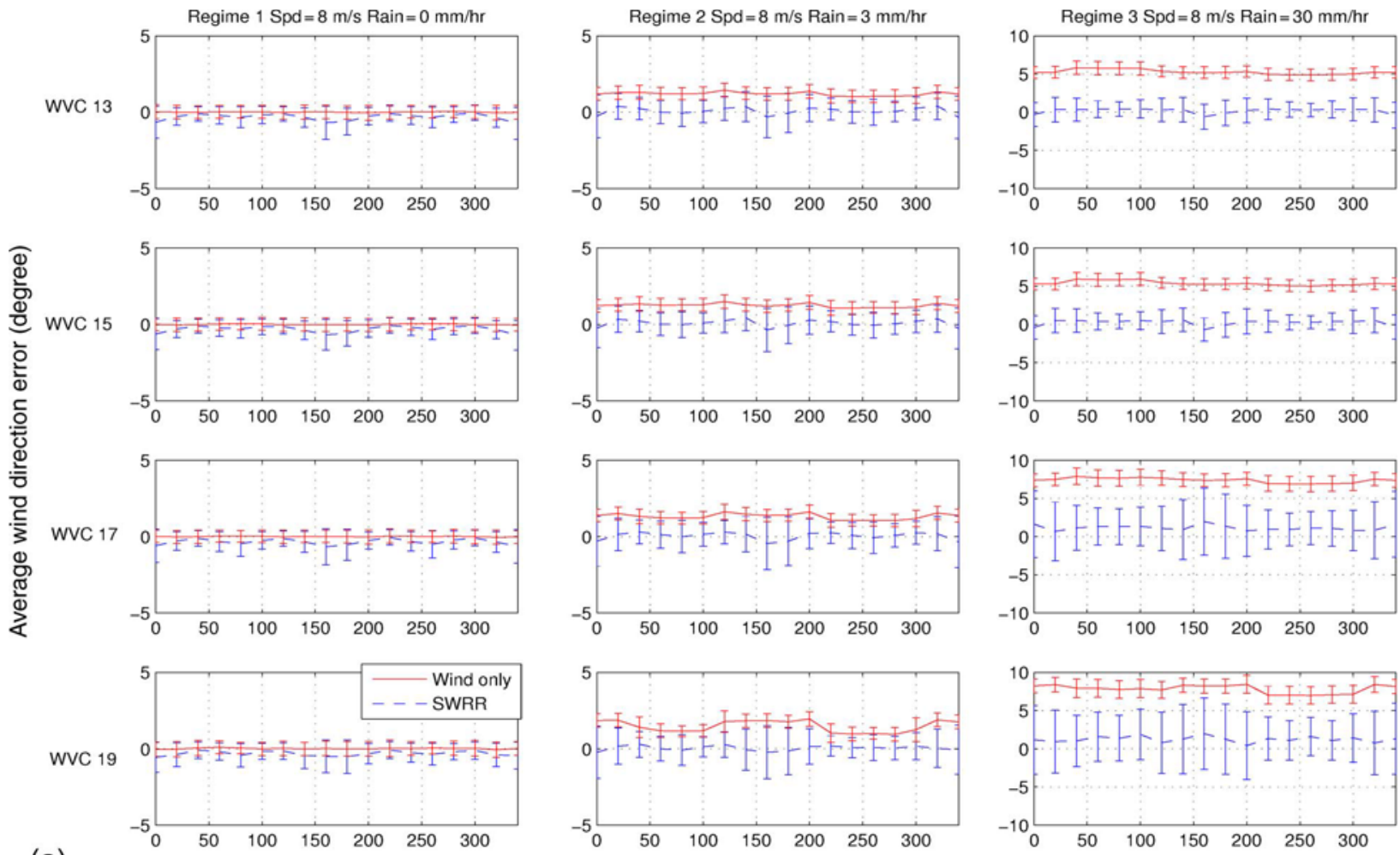

(a)
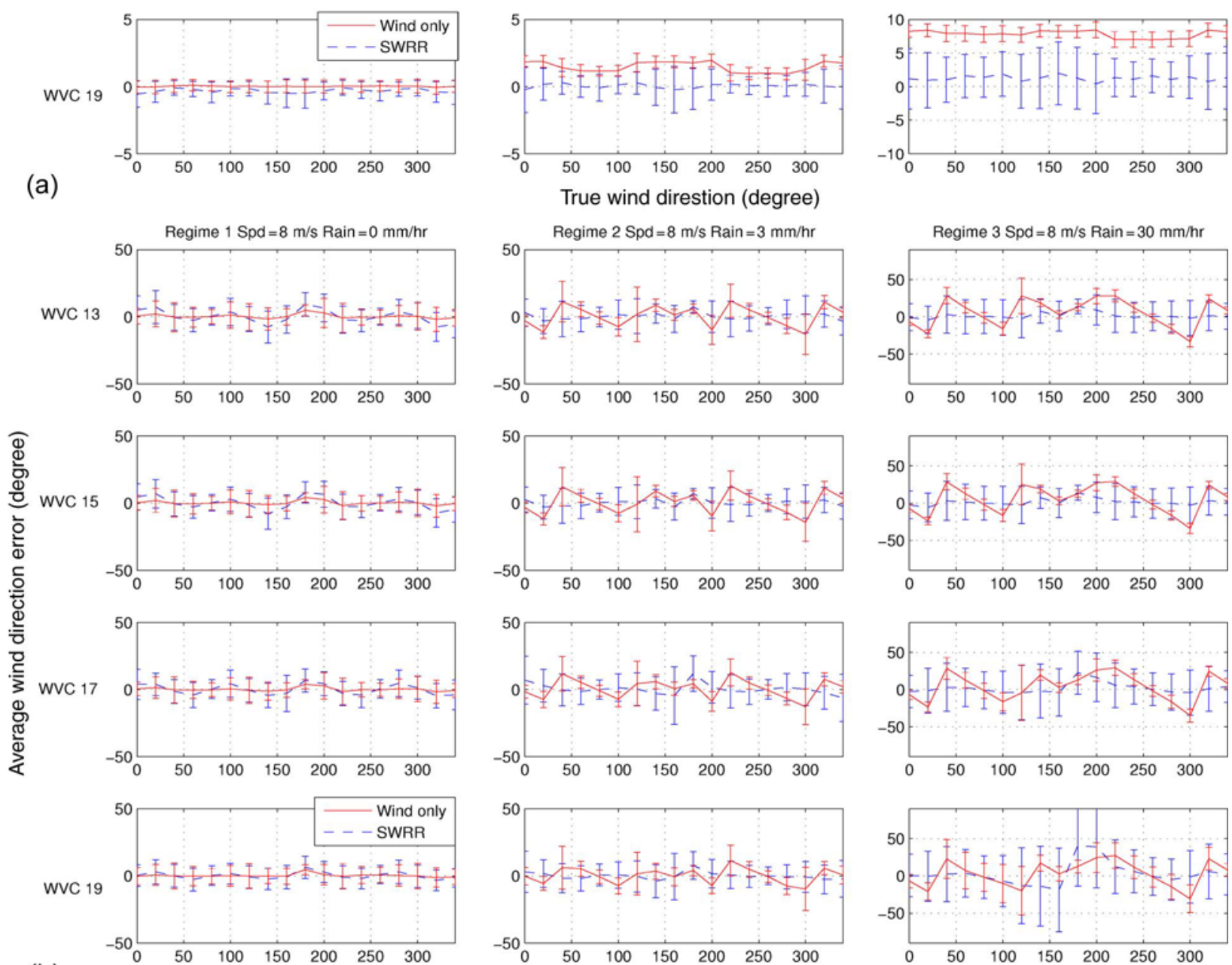

(b)
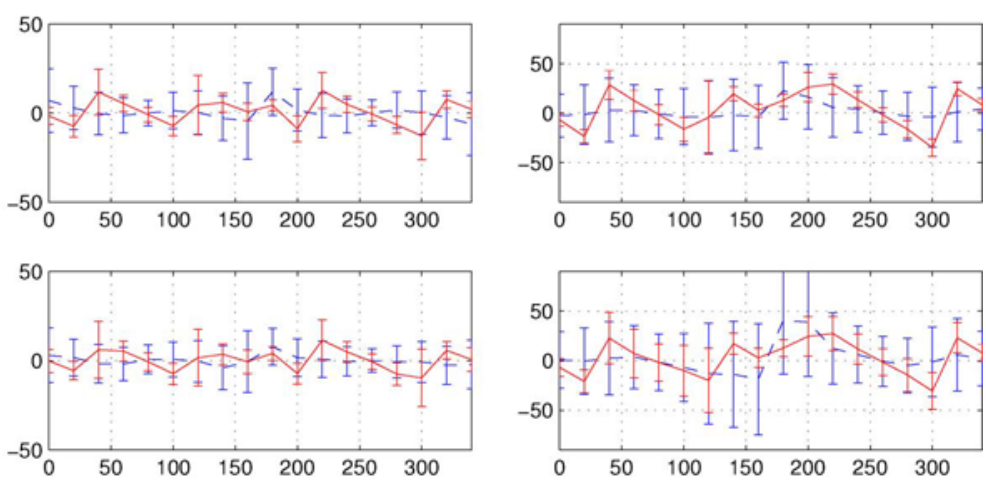

True wind direstion (degree)

Fig. 6. Statistics of wind speed and direction error between retrieved and true (a) wind speeds and (b) directions, respectively, from simulations as a function of the true wind direction for three regime cases with true wind speed of $8 \mathrm{~m} / \mathrm{s}$ for WVCs 13, 15, 17, and 19. In each plot, the results of SWRR are plotted as a dashed line, whereas the results of the wind-only method are plotted as a solid line. Notice the scale differences of the plots. Error bars in the figure represent the STD.

the ocean, whereas the true rain rates are 0,10 , and $30 \mathrm{~mm} / \mathrm{h}$, respectively. In (leftmost column) regime 1, the $\operatorname{Err}_{s}$ of both SWRR and wind-only retrieval are close to zero mean, showing that wind speed estimation is almost unbiased for both methods. The $\operatorname{Err}_{s}$ of SWRR is slightly noisier than that of the windonly retrieval and is slightly biased low, particularly at cross-/ 

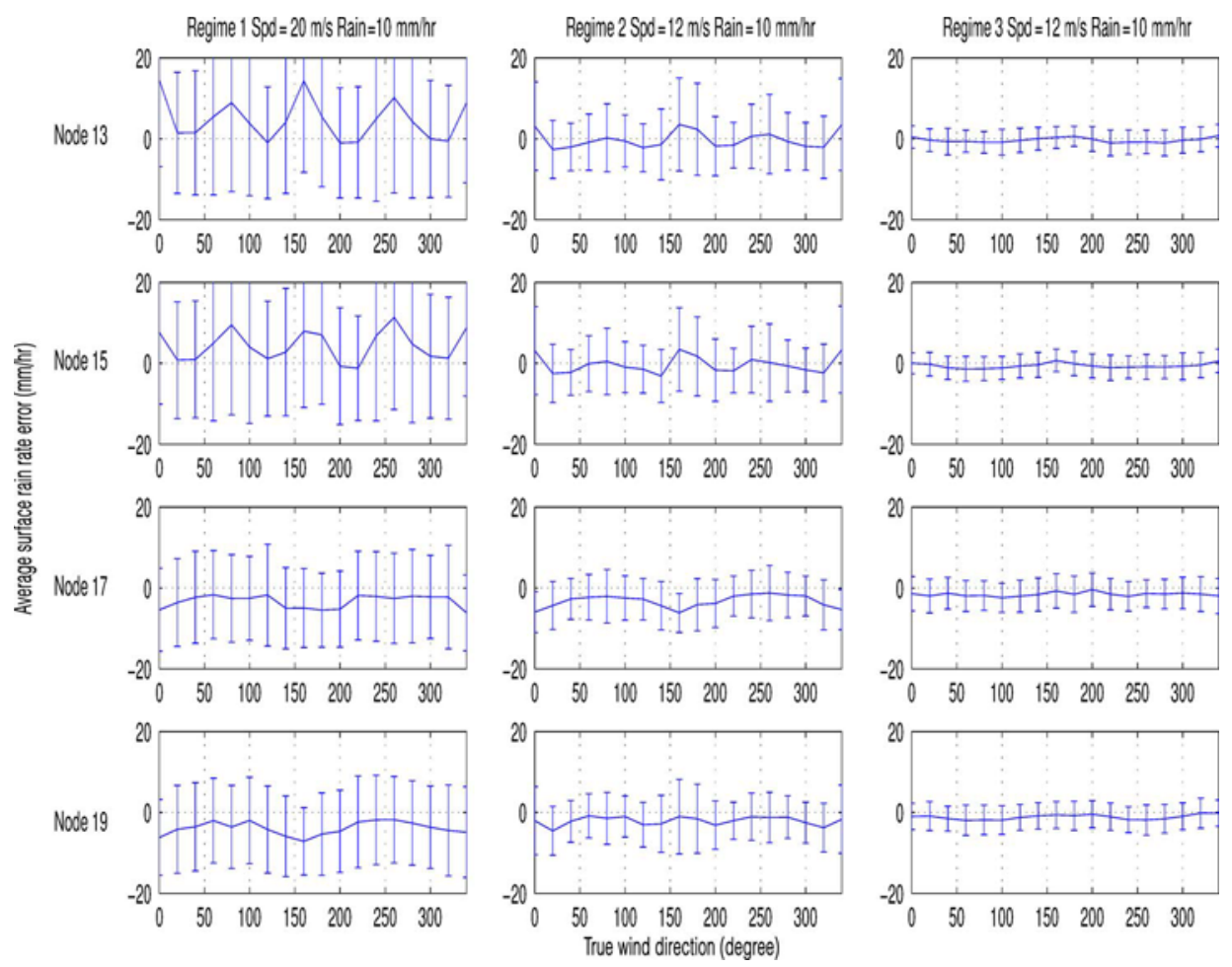

Fig. 7. Statistics of rain rate error between retrieved and true rain rates from simulations as a function of true wind direction for three regime cases with true surface rain rate of $10 \mathrm{~mm} / \mathrm{h}$ for WVCs $13,15,17$, and 19. The line represents the mean error, whereas the error bar represents the STD.

along-swath directions. This is likely due to the introduction of the rain rate to MLE. In regimes 2 and 3, the mean of $\mathrm{Err}_{s}$ for SWRR-retrieved wind speeds is close to zero, whereas $\operatorname{Err}_{s}$ for the wind-only retrieval is biased high, particularly in regime 3 . When rain-induced backscatter highly dominates the backscatter $(\tau>0.9)$, the $\operatorname{Err}_{s}$ for SWRR is slightly biased high, as shown in (rightmost column) regime 3 at WVCs 17 and 19. Under the conditions of high rain domination, the STD of $\operatorname{Err}_{s}$ is relatively high, confirming that it is difficult to accurately estimate the wind speed in this case.

Furthermore, we compare the mean and STD of SWRR and the wind-only error $\operatorname{Err}_{d}=\mathrm{Dir}_{\text {retrieved }}-\mathrm{Dir}_{\text {true }}$ for the same conditions in Fig. 6(b). Similar to the wind speed performance, the $\operatorname{Err}_{d}$ for wind-only retrieval and SWRR are close to zero mean in regime 1, whereas the $\operatorname{Err}_{d}$ for SWRR is somewhat noisier than for the wind-only retrieval. In regime 2 , the zigzagshaped bias in $\operatorname{Err}_{d}$ for wind-only retrieval shows that wind directions are biased toward along-track directions, whereas the $\mathrm{Err}_{d}$ for SWRR is still close to zero mean except for along-track directions. In regime 3 where rain dominates, the wind-only retrieval is significantly biased toward the along-track direction, whereas SWRR performs relatively well at WVCs 13 and 15. At WVCs 17 and 19, high domination by rain degrades the wind direction estimates, particularly at the along-track direction that is close to $180^{\circ}$.

We examine the performance of SWRR-retrieved rain rate by plotting the mean and STD of the difference of the SWRRretrieved and the true rain rate $\operatorname{Err}_{r}=\operatorname{Rain}_{\text {retrieved }}-$ Rain $_{\text {true }}$ as a function of wind direction for a typical case in the three regimes for WVCs 13, 15,17, and 19 in Fig. 7. The true rain rate is $10 \mathrm{~mm} / \mathrm{h}$. In regime 1 where wind dominates, SWRR rain estimation performs poorly. The rain rate cannot be accurately retrieved, particularly for low incidence angle WVCs 13 and 15 where the backscatter measurements are less sensitive to rain. In regime 2 , the retrieved rain rate is close to the zeromean error at WVCs 13 and 15, with a slight bias at along-track directions, whereas $\operatorname{Err}_{r}$ is biased low at WVCs 17 and 19. In regime 3, Err $r$ is close to zero mean and is slightly biased low at WVCs 15, 17, and 19.

Next, in Fig. 8 we demonstrate the speed performance for SWRR and wind-only retrieval by plotting the mean and STD of $\operatorname{Err}_{s}$ as a function of the wind speed under varying rain rate conditions for WVCs 13,15, 17, and 19. Significant biases exist for low-to-moderate wind speeds retrieved by the wind-only method, whereas the SWRR-retrieved wind speed is close to the zero-mean error at low incidence angle WVCs 13 and 15. Under heavy rain rate conditions, SWRR slightly overestimates the wind speed at high incidence angle WVCs 17 and 19. The small bias in the wind speed estimate causes the rain estimate to be biased low in regimes 1 and 2, which is shown in Fig. 9 by plotting the normalized mean rain rate error as a function of the rain ratio $\tau$. The bias in the rain estimate can be corrected by adjusting the wind/rain backscatter model coefficients in the wind retrieval.

\section{VALIDATION}

Next, we compare SWRR and wind-only retrieval using actual ESCAT measurements. Wind vectors retrieved from the two methods are validated by using collocated ECMWF 

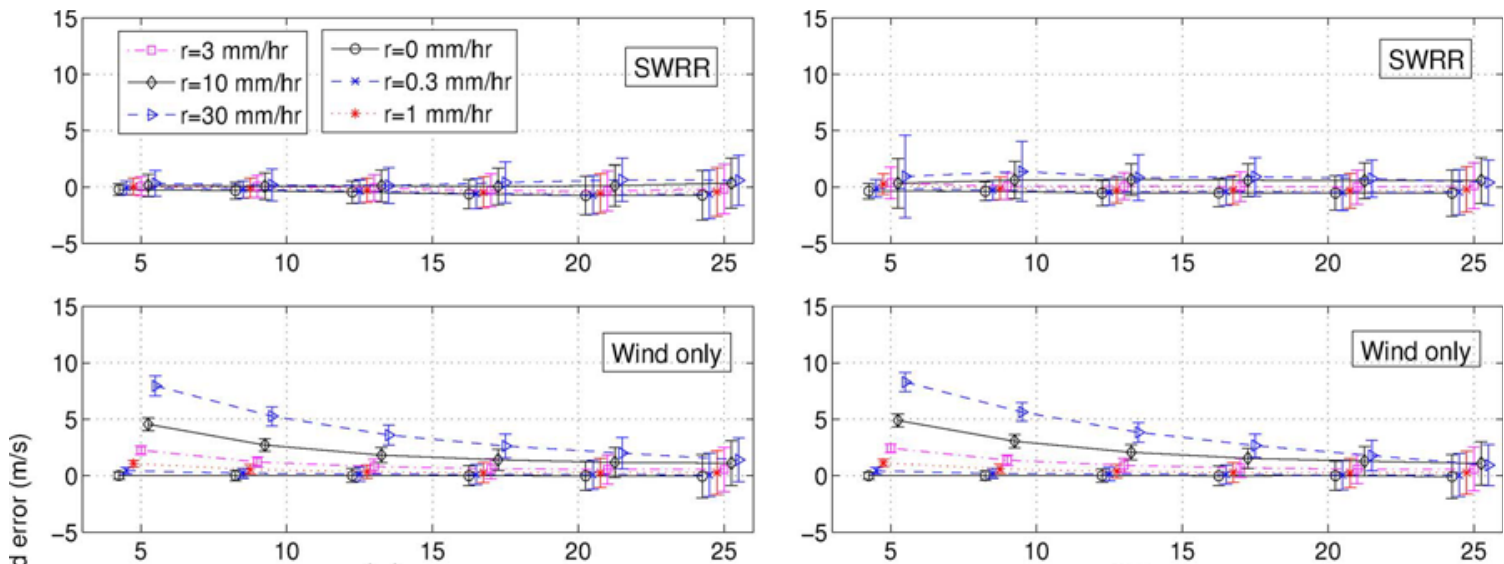

(a) WVC 13
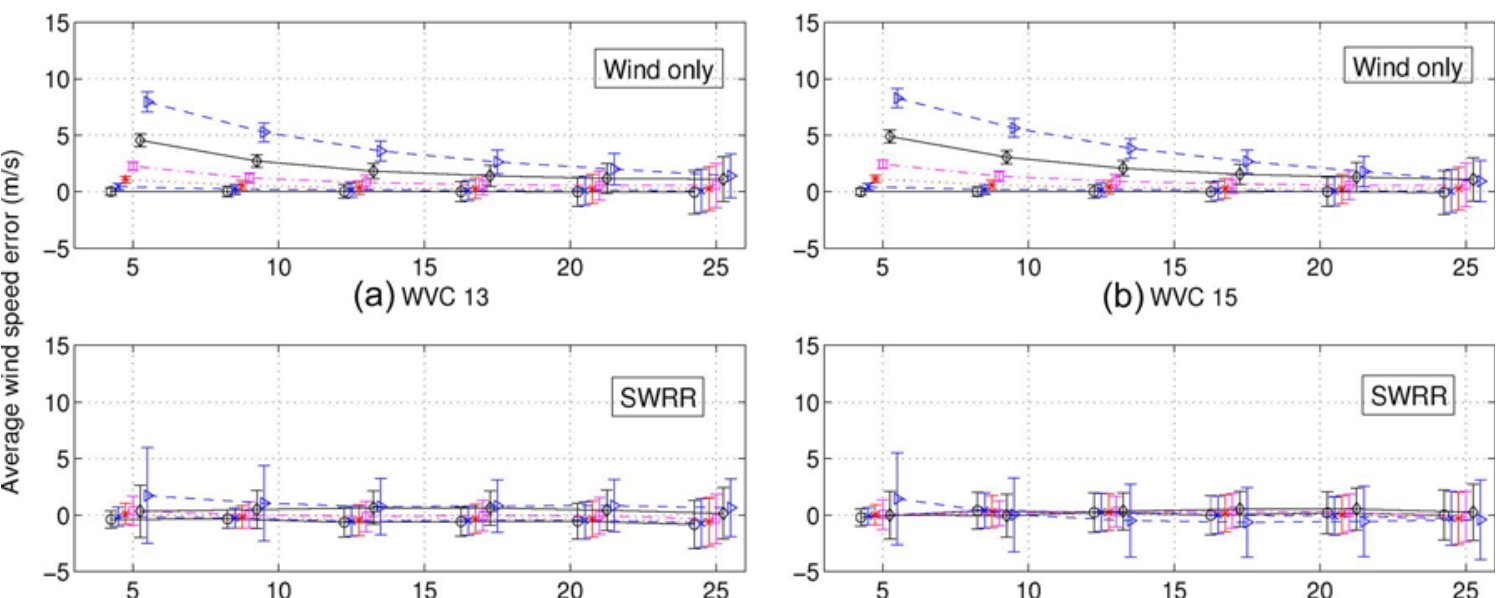

(b) WVC 15

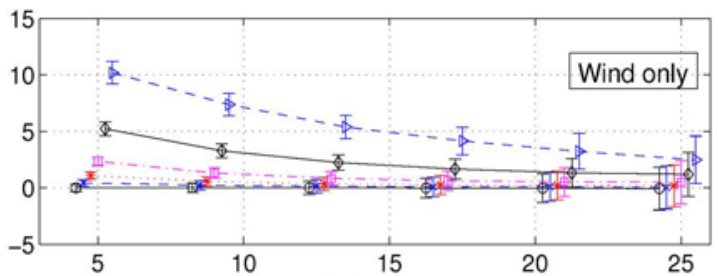

(c) WVC 17
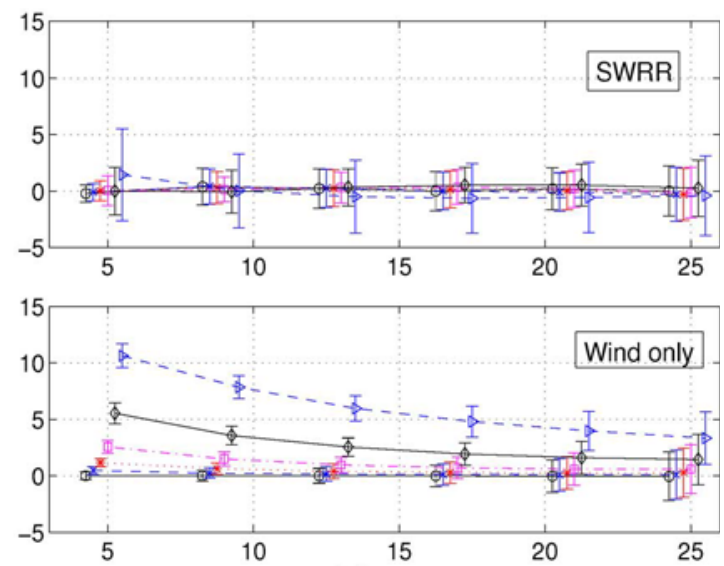

(d) WVC 19

True wind speed $(\mathrm{m} / \mathrm{s})$

Fig. 8. (Lines) Mean and (error bars) STD of wind speed error between retrieved (SWRR and wind-only) and true wind speed from simulations as a function of true wind speed for various rain cases at WVCs (a) 13, (b) 15, (c) 17, and (d) 19. The upper panel shows the results of SWRR, whereas the lower panel shows the wind-only results.

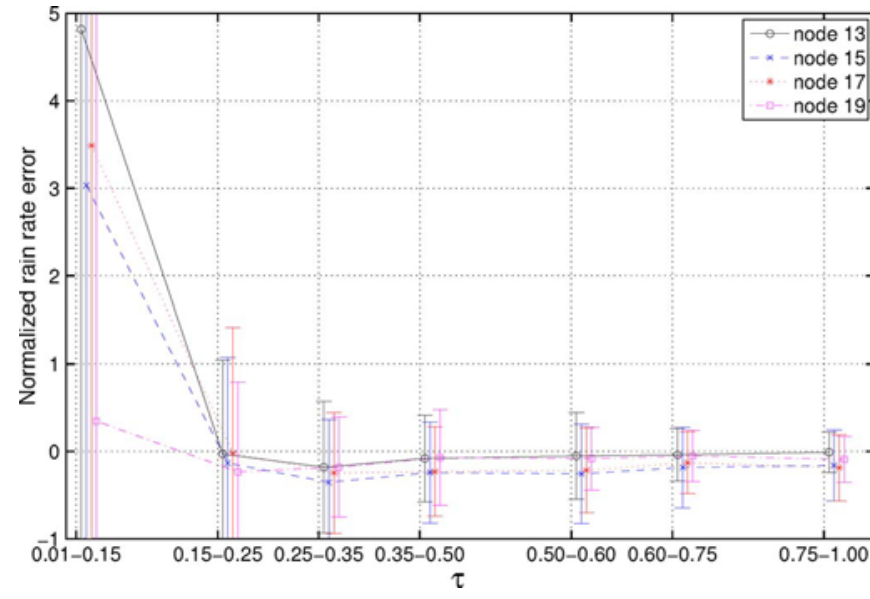

Fig. 9. (Lines) Mean and (error bars) STD of normalized rain error between retrieved and true wind speeds from simulations as a function of the value of $\tau$ at WVCs $13,15,17$, and 19.

wind fields. Fig. 10(a) and (b) shows the scatter density plots of the retrieved (wind-only and SWRR) and ECMWF wind speeds for different rain rate $(R)$ bins. When $R<0.8 \mathrm{~mm} / \mathrm{h}$, the performance of the two methods is similar. The rms of the SWRR-retrieved wind speed is slightly larger than the wind-only retrieval, showing that the wind speed estimates of SWRR are somewhat noisier than the wind-only retrieval. When $R \geq 0.8 \mathrm{~mm} / \mathrm{h}$, the wind-only retrieved wind speed is biased high, whereas the SWRR-retrieved wind speed is close to unbiased. When $R \geq 10 \mathrm{~mm} / \mathrm{h}$, the wind speed of the wind-only retrieval is significantly biased high and has larger rms than SWRR. Thus, SWRR provides more accurate wind estimates than the wind-only retrieval at low to moderate wind speeds when significant rain is present. At most high wind cases where wind dominates, the wind-only speed retrieval is better than SWRR.

To demonstrate a compact comparison of the wind direction retrieval performance of the wind-only retrieval and SWRR, the scatter density plots of the retrieved wind direction (wind-only retrieval and SWRR) and ECMWF predicted wind direction for varying $R$ bins [the same as in Fig. 10(a) and (b)] are shown in Fig. 11(a) and (b). Similar to the wind speed performance, the performance of the wind-only retrieval and SWRR is close for $R<0.8 \mathrm{~mm} / \mathrm{h}$, whereas the SWRR-retrieved wind direction is somewhat noisier. When $R \geq 0.8 \mathrm{~mm} / \mathrm{h}$, the selected wind direction in the wind-only retrieval is biased to along-track directions (the reason for the bias is discussed in Section II-B). 

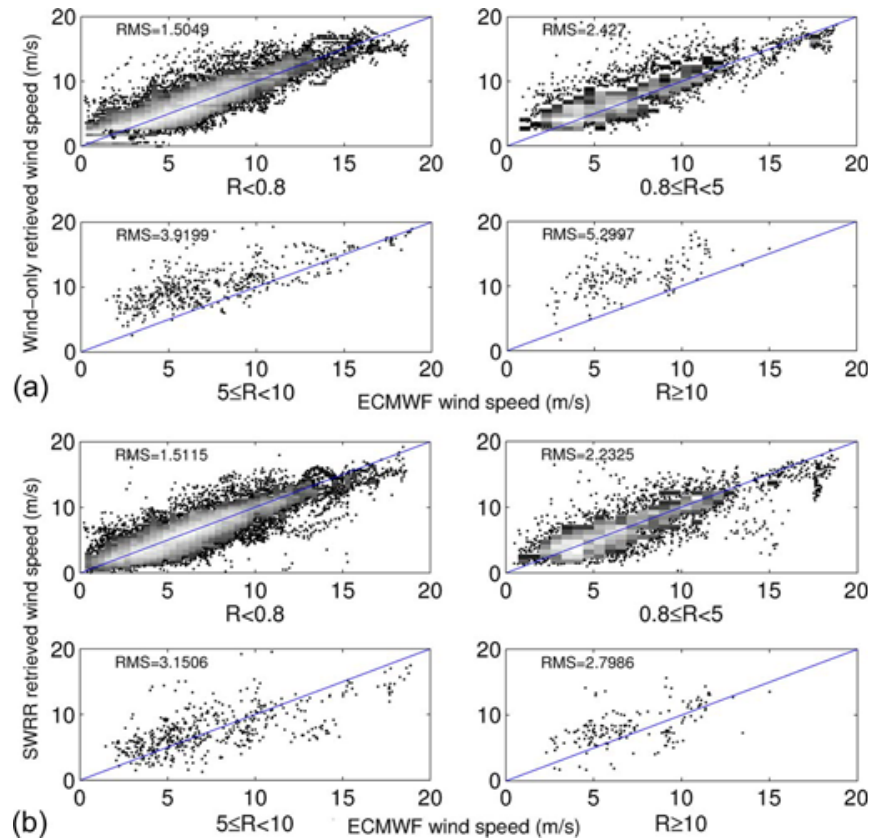

Fig. 10. Scatter density plot of wind-only and SWRR-retrieved wind speeds and collocated ECMWF wind speeds for various rain rate $(R$ is in millimeters per hour) bins. (a) Wind-only retrieved wind speeds versus collocated ECMWF wind speeds for several rain ranges. Bias develops when $R$ is over $5 \mathrm{~mm} / \mathrm{h}$. (b) SWRR-retrieved wind speeds versus collocated ECMWF wind speeds for the same rain ranges. No obvious bias for all $R$ ranges.
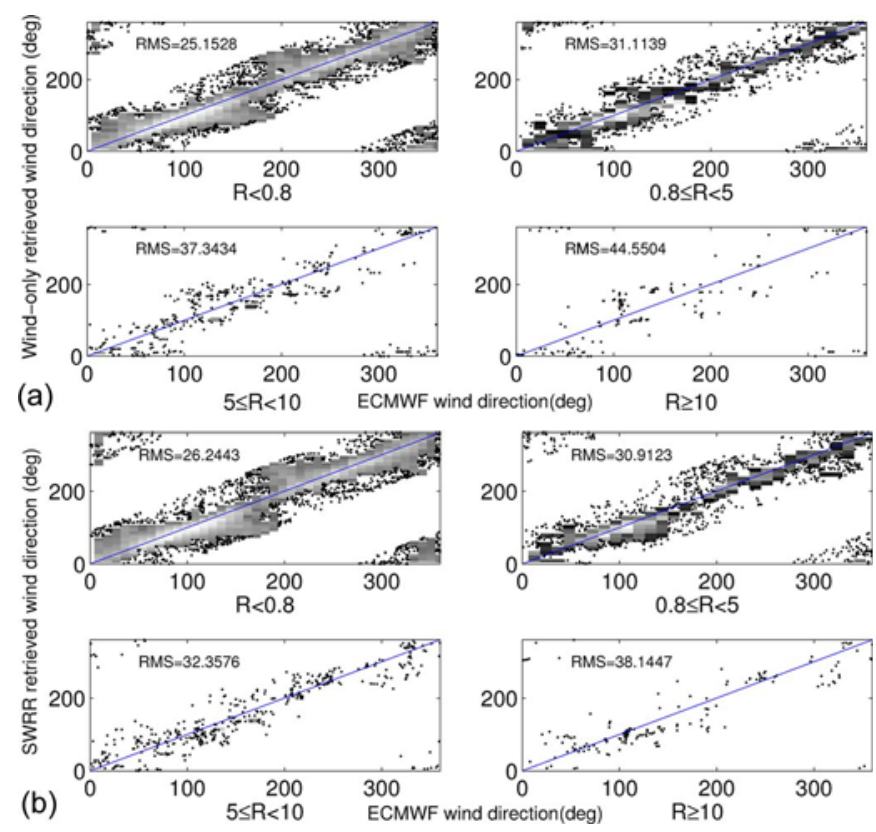

Fig. 11. Scatter density plot of wind-only and SWRR-retrieved wind directions and collocated ECMWF wind directions for various rain rates ( $R$ is in $\mathrm{mm} / \mathrm{h}$ ) bins. (a) Wind-only retrieved wind direction versus collocated ECMWF wind direction. Bias along the along-track direction develops when $R$ is over $5 \mathrm{~mm} / \mathrm{h}$. (b) SWRR-retrieved wind direction versus collocated ECMWF wind direction. No obvious bias exists for all $R$ ranges.

When $R \geq 10 \mathrm{~mm} / \mathrm{h}$, the wind direction of the wind-only retrieval is significantly biased to along-track directions and has larger rms than SWRR. For $R \geq 10 \mathrm{~mm} / \mathrm{h}$, the SWRR-retrieved wind direction is close to unbiased but is noisy.
To validate the surface rain rates retrieved from SWRR, we show a scatter density plot of SWRR-retrieved and TRMM PR surface rain rates collocated within \pm 15 min in Fig. 12(a). Because the plot is in log-log space, zero rain rates in either of the SWRR or TRMM PR data sets are not displayed. Of the rain rates that are zero in either of the two data sets, over $95 \%$ have relatively small rain rates $(\leq 3 \mathrm{~mm} / \mathrm{h})$ in the other data set. The SWRR- and TRMM PR-estimated rain rates have a relatively high correlation, although the SWRR rain rates have considerable scatter compared with the TRMM PR rain rates. The SWRR rain rate is biased high for low rain rates (approximately $\leq 0.8 \mathrm{~mm} / \mathrm{h}$ ) and biased low for moderate-to-high rain rates, which is consistent with the results of the aforementioned simulation. Considering that the temporal variability of rain events can significantly contribute to the variability between the SWRR- and TRMM PR-derived rain rates, we examine the relationship between the two rain rates within \pm 2 min in Fig. 12(b). Here, the SWRR-retrieved rain rate is highly correlated with TRMM PR rain rates, with a correlation coefficient of 0.89 and rms of 2.024, demonstrating the relatively high accuracy of SWRR-retrieved rain. In addition to a scatter-plot between the two rain rates, a histogram of rain rates can be used as a statistical comparison method to validate SWRR-retrieved rain. Fig. 12(c) shows the histograms of the collocated rain rates estimated by both (solid line) SWRR and (dashed line) TRMM PR, where the histograms of the two rain rates match relatively well for moderate-to-high rain rates and the curve is biased low for low rain rates.

Through validation, SWRR is shown to significantly improve the wind estimates in regimes where the wind- and raininduced backscatters are on the same order. When the raininduced backscatter dominates the total backscatter, SWRR wind estimates are noisy but almost unbiased. SWRR-retrieved rain rates have relatively high accuracy for moderate-to-high rain rates. SWRR-retrieved rain rates are somewhat biased but can be corrected. Unfortunately, considering that ESCAT is not particularly designed for rain detection, the introduction of rain rate into wind retrieval makes SWRR wind estimates noisier than the wind-only retrieval, and rain rate estimates are inaccurate in regimes where wind-induced backscatter dominates the total backscatter. Considering that the wind-only retrieval method performs well in regime 1, SWRR is less useful here. Furthermore, due to the geometry of ESCAT and the limitations of MLE, SWRR does not perform well at along-track wind directions, which are where SWRR often misidentifies wind and rain. Thus, it is most useful to implement SWRR only in raining areas and use wind-only retrieval in rain-free areas. SWRR-retrieved wind/rain can be used when the rain ratio $\tau$ is greater than a specific threshold. MLE-based QC methods [11] can be used for rain thresholding to improve the accuracy of wind/rain estimates.

\section{CAse Study}

To demonstrate the performance of SWRR under different circumstances, we examine a typical case of SWRR-retrieved wind/rain fields. The case shows a storm over the Pacific Ocean at about latitude $35^{\circ}$ and longitude $215^{\circ}$ at UTC 08:13:47 

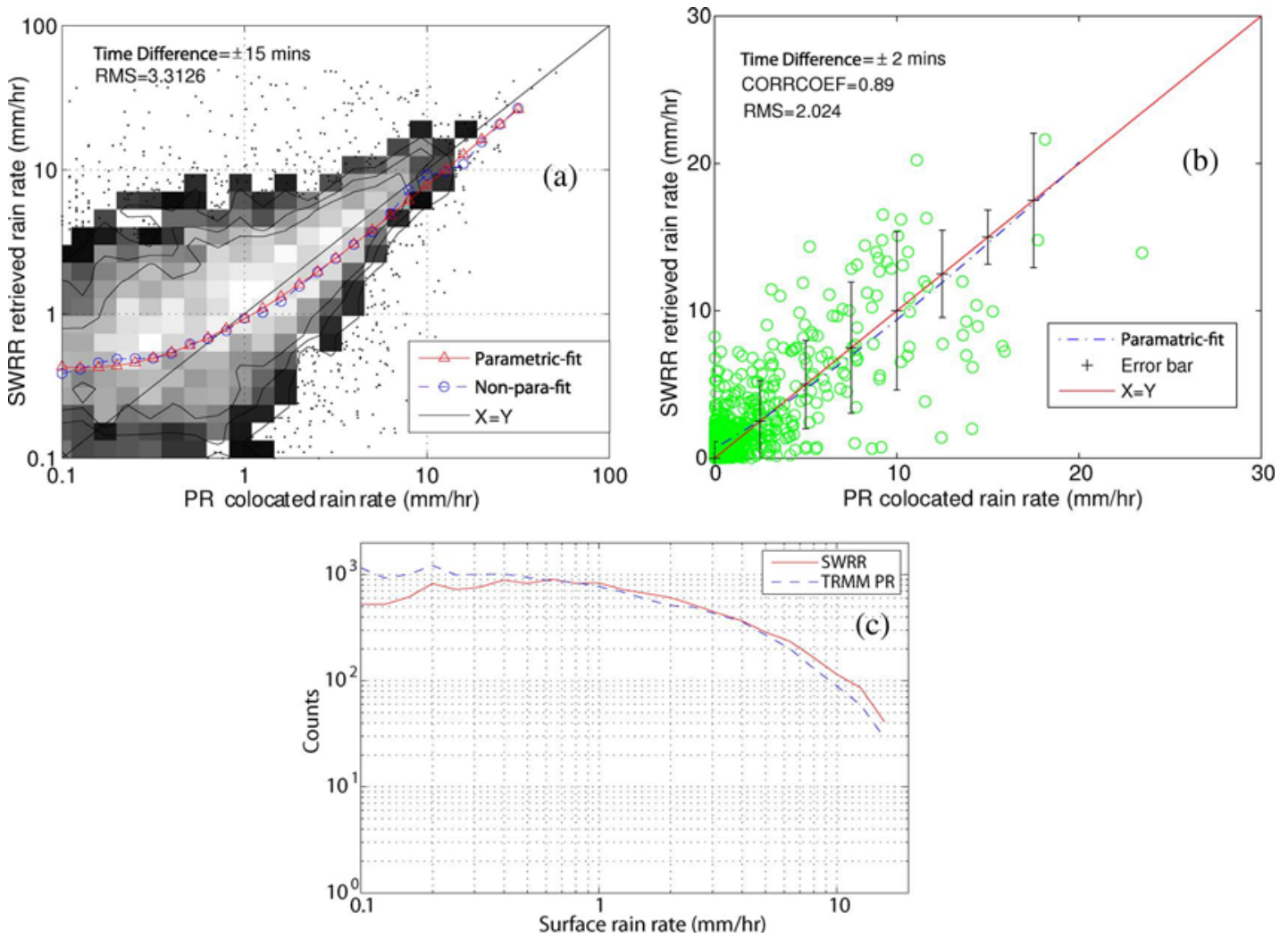

Fig. 12. Scatter density plot of SWRR rain rates versus TRMM PR antenna-weighted rain rates within (a) \pm 15 min in $\log -\log$ space and within (b) \pm 2 min in normal space. Nonparametric and best quadratic fits to TRMM PR rain rate in log-log space in (a) and best quadratic fit in normal space in (b) are also shown. Error bars represent the STD. (c) Histograms of SWRR and TRMM PR rain rates.

on December 25, 2000. We compare the SWRR-retrieved wind/rain, wind-only retrieved wind, ECMWF winds, and collocated TRMM PR rain rates in Fig. 13.

In Fig. 13 (b), the wind-only wind fields exhibit many raincontaminated features. In the heavy rain area at the lower right, dramatic wind speed inconsistency and along-track wind directions are present, indicating severe rain contamination. In Fig. 13 (a), SWRR-retrieved wind vectors in the raining area are more consistent with nearby rain-free WVCs. SWRRretrieved wind vectors also agree with the ECMWF wind fields in Fig. 13 (c) much better than the wind-only retrieved wind vectors, showing that SWRR significantly improves the wind estimates in this case. Comparing the rain fields in panels (d) and (e) of Fig. 13, we find similar spatial rain patterns in the SWRR-retrieved and TRMM PR antenna-weighted rain. Although SWRR somewhat overestimates rain rates in this case, the SWRR-retrieved rain rate is highly correlated with the TRMM PR antenna-weighted rain rates.

\section{CONCLUSION}

Using collocated TRMM PR, ESCAT on ERS 1/2, and ECMWF data, rain effects on ESCAT wind-only retrieval are evaluated and analyzed. Wind speed retrieved by wind-only retrieval is biased high due to the rain-induced backscatter. Rain contamination causes the wind direction estimates to be biased toward the along-track directions under heavy rain conditions no matter what the true wind direction. Rain effects on the wind-only retrieval varies with incidence angles. The higher the incidence angle, the more significant the rain impact is on the wind-only retrieval.

To compensate for rain-induced backscatter, we develop a SWRR method for a C-band scatterometer for an incidence angle $>40^{\circ}$ based on a wind/rain backscatter model. Through simulation and validation with collocated ESCAT, TRMM PR, and ECMWF data, we find that SWRR can significantly improve wind speed and wind direction estimates in regimes 2 and 3, where rain and wind-induced backscatter are on the same order or rain dominates the total backscatter. In addition, SWRR can retrieve rain rate from ESCAT measurements. The rain estimates have relatively high accuracy in regimes 2 and 3. In regime 1, where wind-induced backscatter dominates the total backscatter, the accuracy of SWRR-retrieved rain rates is degraded, and spurious rain rates may be derived. Due to limitations in the MLE, SWRR does not perform well when the wind direction aligns in along-track directions. In regime 1 (which includes most high wind cases), the performance of SWRR is close to that of the wind-only retrieval, although it is somewhat noisier due to the introduction of a new parameter (rain) into the retrieval. Therefore, SWRR wind/rain should be used only when the rain ratio $\tau$ is over a specific threshold.

Although for ESCAT, only about $1.5 \%$ of all the collocated measurements are affected by significant rain [1], SWRR can enable the accurate wind retrieval of a high percentage of raincontaminated measurements that would otherwise be unusable. 

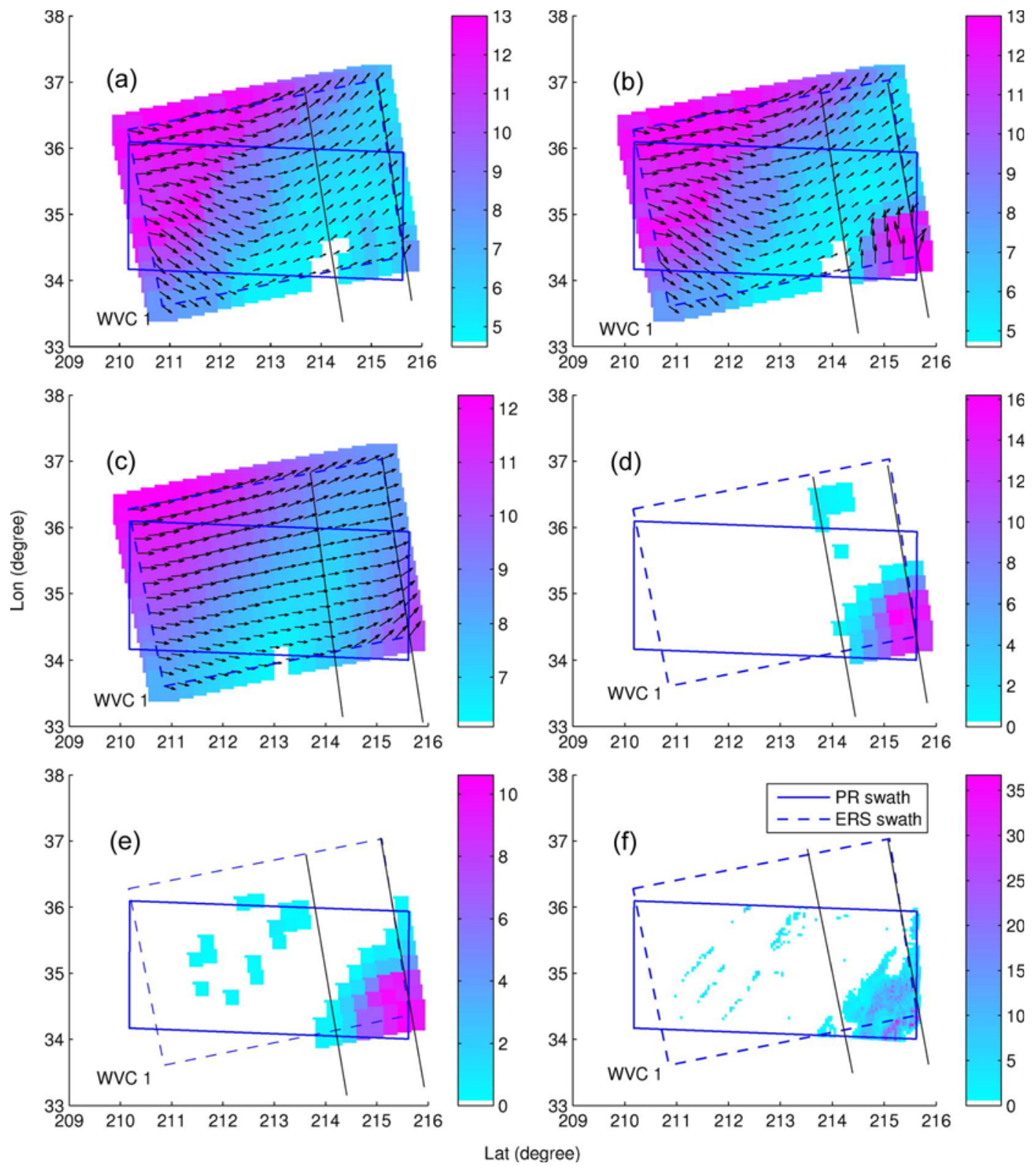

Fig. 13. Example of an SWRR-retrieved wind/rain field. (a)-(c) show SWRR-retrieved, wind-only retrieved, and ECMWF wind vectors with corresponding wind speeds plotted as background. (d)-(f) show SWRR-retrieved rain, TRMM PR antenna-weighted rain, and TRMM PR retrieved rain. The TRMM PR swath is outlined by a blue solid line, whereas the ESCAT swath is outlined by a dashed line. WVCs 13-19, which are where SWRR is performed, are outlined by two black solid lines.

Considering that ASCAT on MetOP is expected to be more sensitive to rain due to its higher incidence angle, SWRR can also benefit ASCAT wind retrieval.

\section{ACKNOWLEDGMENT}

The authors would like to thank the Physical Oceanography Distributed Active Archive Centre for providing the ESCAT data, the TRMM Science Data and Information System for offering TRMM PR data, and the reviewers for their comments.

\section{REFERENCES}

[1] C. Nie and D. G. Long, "A C-band wind/rain backscatter model," IEEE Trans. Geosci. Remote Sens., vol. 45, no. 3, pp. 621-631, Mar. 2007.

[2] R. F. Contreras and W. J. Plant, "Surface effect of rain on microwave backscatter from the ocean: Measurements and modeling," J. Geophys. Res., vol. 111, no. C8, p. C08 019, Aug. 2006.
[3] C. Melsheimer, W. Alpers, and M. Gade, "Simultaneous observations of rain cells over the ocean by the synthetic aperture radar aboard the ERS satellites and by surface-based weather radars," J. Geophys. Res., vol. 106, no. C3, pp. 4665-4677, 2001.

[4] M. Portabella and A. Stoffelen, "Rain detection and quality control of SeaWinds," J. Atmos. Ocean. Technol., vol. 18, no. 7, pp. 1171-1183, Jul. 2001.

[5] M. Portabella and A. Stoffelen, "A comparison of KNMI quality control and JPL rain flag for SeaWinds," Can. J. Remote Sens., vol. 28, no. 3, pp. 424-430, Jun. 2002.

[6] J. Figa and A. Stoffelen, "On the assimilation of Ku-band scatterometer winds for weather analysis and forecasting," IEEE Trans. Geosci. Remote Sens., vol. 38, no. 4, pp. 1893-1902, Jul. 2000.

[7] D. W. Draper and D. G. Long, "Evaluating the effect of rain on SeaWinds scatterometer measurements," J. Geophys. Res., vol. 109, no. C12, pp. C02 005.1-C02 005.12, Feb. 2004.

[8] JPL, QuickSCAT Science Data Product User's Manual, 2001, Pasadena, CA: California Inst. Technol., Jet Propulsion Lab.

[9] D. G. Long and M. W. Spencer, "Radar backscatter measurement accuracy for a spaceborne pencil-beam wind scatterometer with transmit 
modulation," IEEE Trans. Geosci. Remote Sens., vol. 35, no. 1, pp. 102114, Jan. 1997.

[10] T. E. Oliphant and D. G. Long, "Accuracy of scatterometer-derived winds using the Cramer-Rao bound," IEEE Trans. Geosci. Remote Sens., vol. 37, no. 6, pp. 2642-2652, Nov. 1999.

[11] A. C. M. Stoffelen and D. L. T. Anderson, "Scatterometer data interpretation: Measurement space and inversion," J. Atmos. Ocean. Technol., vol. 14, no. 6, pp. 1298-1313, Dec. 1997.

[12] J. N. Huddleston and B. W. Stiles, "A multi-dimensional histogram rain flagging technique for Sea Winds on QuickSCAT," in Proc. Int. Geosci. Remote Sens. Symp., Honolulu, HI, 2000, vol. 3, pp. 1232-1234.

[13] D. W. Draper and D. G. Long, "Assessing the quality of SeaWinds rain measurements," IEEE Trans. Geosci. Remote Sens., vol. 42, no. 7, pp. 1424-1432, Jul. 2004.

[14] D. W. Draper and D. G. Long, "Simultaneous wind and rain retrieval using SeaWinds data," IEEE Trans. Geosci. Remote Sens., vol. 42, no. 7, pp. 1411-1423, Jul. 2004.

[15] M. Portabella and A. Stoffelen, "Scatterometer backscatter uncertainty due to wind variability," IEEE Trans. Geosci. Remote Sens., vol. 44, no. 11, pp. 3356-3362, Nov. 2006

[16] H. Hersbach, A. Stoffelen, and S. de Haan, "An improved C-band scatterometer ocean geophysical model function: CMOD5," J. Geophys. Res., vol. 112, no. C3, p. C03 006, 2007.

[17] C. Kummerow et al., "The status of the Tropical Rainfall Measuring Mission (TRMM) after 2 years in orbit," J. Appl. Meteorol., vol. 39, no. 12 , pp. 1965-1982, Dec. 2000.

[18] C. Kummerow, W. Barnesa, T. Kozub, J. Shiuec, and J. Simpson, "The Tropical Rainfall Measuring Mission (TRMM) sensor package," J. Atmos. Ocean. Technol., vol. 15, no. 3, pp. 809-817, Jun. 1998.

[19] D. Halpern, E. Harrison, B. Hinton, R. Long, M. Mathur, J. O'Brien, S. Payne, S. Pazan, and J. Sadler, "Intercomparison of tropical Pacific mean November 1979 surface wind fields," Dept. Meteorol. Phys. Oceanogr., Massachusetts Inst. Technol., p. 40, 1982. Tech. Rep.

[20] D. Halpern, A. Hollingsworth, and F. Wentz, "ECMWF and SSM/I global surface wind speeds," J. Atmos. Ocean. Technol., vol. 11, no. 3, pp. 779-788, Jun. 1994.

[21] F. Rabier, H. Jarvinen, E. Klinker, J. F. Mahfouf, and A. Simmons, "The ECMWF operational implementation of four-dimensional variational assimilation. Part I: Experimental results with simplified physics," $Q$. J. R. Meteorol. Soc., vol. 126, pp. 1143-1170, 2000.

[22] B. W. Stiles and S. Yueh, "Impact of rain on spaceborne Ku-band wind scatterometer data," IEEE Trans. Geosci. Remote Sens., vol. 40, no. 9, pp. 1973-1983, Sep. 2002.

[23] M. W. Spencer and M. Shimada, "Effect of rain on Ku-band scatterometer wind measurements," in Proc. Int. Geosci. Remote Sens. Symp., 1991, vol. 3, pp. $1285-1288$.
[24] S. J. Shaffer, R. S. Dunbar, S. V. Hsiao, and D. G. Long, "A median-filterbased ambiguity removal algorithm for NSCAT," IEEE Trans. Geosci. Remote Sens., vol. 29, no. 1, pp. 167-174, Jan. 1991.

[25] J. Goldhirsh, "Rain cell size statistics as a function of rain rate for attenuation modeling," IEEE Trans. Antennas Propag., vol. AP-31, no. 5, pp. 799-801, Sep. 1983.

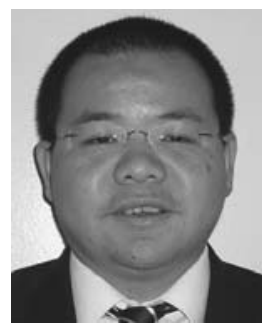

Congling Nie received the B.S. degree in electrical engineering from South China University of Technology, Guangzhou, China, in 1995 and the Ph.D. degree in electrical engineering from Brigham Young University (BYU), Provo, UT, in 2008.

From 1995 to 2003, he was with the Meteorological Center of Central and South China Air Traffic Management Bureau, where he developed weather radar applications for air traffic control. Since 2003, he has been with the Microwave Earth Remote Sensing Research Group, Department of Electrical and Computer Engineering, BYU, in 2003. His current research interests are rain effects on microwave backscatter from ocean surfaces and scatterometer wind retrieval.

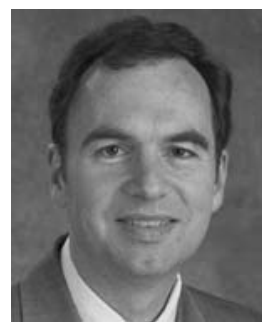

David G. Long (S'80-SM'98-F'08) received the $\mathrm{Ph} . \mathrm{D}$. degree in electrical engineering from the University of Southern California, Los Angeles, in 1989.

From 1983 to 1990 he worked for NASA's Jet Propulsion Laboratory (JPL), where he developed advanced radar remote sensing systems. While at JPL, he was the Project Engineer on the NASA Scatterometer (NSCAT) project, which flew from 1996 to 1997 . He also managed the SCANSCAT project, which is the precursor to SeaWinds, which was launched in 1999 and 2002. He is currently a Professor with the Department of Electrical and Computer Engineering, Brigham Young University (BYU), Provo, UT, where he teaches upper division and graduate courses in communications, microwave remote sensing, radar, and signal processing, and where he is the director of the BYU Center for Remote Sensing. He is the principle investigator on several NASA-sponsored research projects in remote sensing. He has over 280 publications in signal processing and radar scatterometry. His research interests include microwave remote sensing, radar theory, space-based sensing, estimation theory, signal processing, and mesoscale atmospheric dynamics.

Dr. Long has received the NASA Certificate of Recognition several times. $\mathrm{He}$ is an associate editor for the IEEE Geoscience and Remote Sensing LETTERS. 